\title{
Molecular mechanism of ACAD9 in mitochondrial respiratory complex 1 assembly
}

Chuanwu Xia ${ }^{1}$, Baoying Lou ${ }^{1}$, Zhuji Fu ${ }^{1}$, Al-Walid Mohsen ${ }^{2}$, Jerry Vockley ${ }^{2}$, and Jung-Ja P. Kim ${ }^{1}$

${ }^{1}$ Department of Biochemistry, Medical College of Wisconsin, Milwaukee, Wisconsin, 53226, USA ${ }^{2}$ Department of Pediatrics, University of Pittsburgh School of Medicine, University of Pittsburgh, Children's Hospital of Pittsburgh of UPMC, Pittsburgh, PA 15224, USA

\section{Abstract}

ACAD9 belongs to the acyl-CoA dehydrogenase family, which catalyzes the $\alpha-\beta$ dehydrogenation of fatty acyl-CoA thioesters. Thus, it is involved in fatty acid $\beta$-oxidation (FAO). However, it is now known that the primary function of ACAD9 is as an essential chaperone for mitochondrial respiratory complex 1 assembly. ACAD9 interacts with ECSIT and NDUFAF1, forming the mitochondrial complex 1 assembly (MCIA) complex. Although the role of MCIA in the complex 1 assembly pathway is well studied, little is known about the molecular mechanism of the interactions among these three assembly factors. Our current studies reveal that when ECSIT interacts with ACAD9, the flavoenzyme loses the FAD cofactor and consequently loses its FAO activity, demonstrating that the two roles of ACAD9 are not compatible. ACAD9 binds to the carboxy-terminal half (C-ECSIT), and NDUFAF1 binds to the amino-terminal half of ECSIT. Although the binary complex of ACAD9 with ECSIT or with C-ECSIT is unstable and aggregates easily, the ternary complex of ACAD9-ECSIT-NDUFAF1 (i.e., the MCIA complex) is soluble and extremely stable. Molecular modeling and SAXS studies of the MCIA complex identified the possible interaction sites between the three assembly factors and binding sites for other assembly factors, including complex 1 subunits. Furthermore, we have mapped over 40 currently known pathogenic mutation sites onto the homology-modeled ACAD9 structure, giving us the structural basis for their involvement in diseases that result from complex 1 deficiency.

\section{Introduction}

ACAD9 was first identified, by large scale random sequencing, as a member of the acyl-CoA dehydrogenase family that catalyzes the $\alpha-\beta$ dehydrogenation of fatty acyl-CoA thioesters, and is involved in mitochondrial fatty acid $\beta$-oxidation (FAO) (1). The enzyme is highly homologous to very long chain acyl-CoA dehydrogenase (VLCAD) and is capable of carrying out the $\alpha-\beta$ dehydrogenation of palmitoyl-CoA (1). Further characterizations showed that, like VLCAD, ACAD9 has maximal activity with long chain unsaturated acyl-CoAs (2). However, following a report that ACAD9-deficient patients demonstrated fatty acid oxidation dysfunction (3), ACAD9 was shown to be an essential assembly factor for mitochondrial oxidative phosphorylation complex $1(\mathrm{C} 1)$ and to bind two known complex 1 assembly 
factors, ECSIT and NDUFAF1 (4). Furthermore, DNA sequencing of C1-deficient patients revealed mutations in the ACAD9 gene, but showed no disturbance in long chain fatty acid oxidation (4). However, Nouws et al. showed later that while ACAD9's primary cellular function is that of a C1 assembly factor, it also participates in mitochondrial fatty acid oxidation when necessary (5). Schiff et al. also showed that ACAD9 plays a physiological role in long-chain fatty acid oxidation, independent of its role as a C1 assembly factor, by using ACAD9-knockdown HEK293 and mouse fibroblast cell lines (6). In HEK293 cells, whole cellular mitochondrial palmitate oxidation decreased by 35-40 \% when ACAD9 was knocked down, while ECSIT, a binding partner in ACAD9-mediated C1 assembly, was also absent. When both LCAD and VLCAD were knocked down, the mouse fibroblast cells displayed 50\% residual long chain FAO activity, consistent with ACAD9's physiologic role in FAO for these cells.

Like ACAD9, many assembly factors also have other functions; Ecsit (Evolutionarily Conserved Signaling Intermediate in Toll pathway) in the cytoplasm is a signaling protein in two pathways, the Toll pathway and the BMP pathway (7) (8). Similarly, NDUFAF7 functions as a protein arginine methyltransferase in the nucleus (9). However, ACAD9 is unique, as ACAD9 performs both functions in the same organelle, the mitochondria.

$\mathrm{C} 1$ (NADH-ubiquinone oxidoreductase) is the first enzyme complex of the mitochondrial respiratory chain. It oxidizes NADH to yield electrons facilitating the translocation of protons across the mitochondrial inner membrane and the generation of a proton gradient. Eukaryotic complex I consists of 14 conserved subunits that are homologous to the bacterial subunits and more than 26 accessory subunits (10). In mammals, complex I consists of 45 subunits with a molecular mass of 980kDa that must be assembled correctly to form the properly functioning mature complex. It is one of the largest membrane protein assemblies known so far, and its dysfunction is the most common oxidative phosphorylation (OXPHOS) disorder in humans(11). Thus, C1requires various assembly factors functioning in a coordinated process $(12,13)$. Currently, at least 15 assembly factors have been identified (14-16). However, the exact mechanisms by which these proteins facilitate and regulate the complex I assembly remain to be elucidated.

In the current paper, we have cloned and expressed human ACAD9, ECSIT, and NDUFAF1 and characterized the biochemical and biophysical properties of their binary and ternary complexes as well as the three individual proteins. The results reveal the structural and mechanistic bases for the role of ACAD9 in complex 1 assembly and enable us to propose the mechanism of the regulation of its dual activities in mitochondrial complex 1 assembly and the fatty acid $\beta$-oxidation pathway.

\section{Experimental Procedures}




\section{Materials}

Palmitoyl-coenzyme A was purchased from Avanti Polar Lipids, Inc. (Alabaster, AL). Restriction enzymes, DNA polymerases, ligase, DNA, and protein markers were purchased from Thermo Fisher Scientific (Rockford, IL). Ni-NTA resin was purchased from Clontech Laboratories (Mountain View, CA). All other chemicals were purchased from Sigma Aldrich (St. Louis, MO) or RPI Corp. (Mount Prospect, IL).

\section{Cloning, Expression, and Purification of Human ACAD9, ECSIT, and NDUFAF1}

Cloning of human ACAD9 in pET21a vector (ACAD9_pET21a without a tag) has been previously described (2). Also, to facilitate purification, a C-terminal Histag (-GSHHHHHH) was introduced to the non-tagged clone (referred to as ACAD9-His___pET21) by using PCR and two primers (T7 promoter 5'TAATACGACTCACTATAGGG-3' as 5' sense primer and 5'-CCCGGC GTCGAC TCA GTG ATG ATG-GTG GTG ATG GCT CCC GCA GGT GCG GTC CAG AGG-3' as 3' sense primer). All ACAD9 mutants used in this study, including Arg469Trp, Arg518His, Arg532Trp, and Glu426Gln, were cloned in ACAD9-His_6_pET21b vector using the QuickChange Site-Directed Mutagenesis Kit as previously reported (6). Two mutants with a shortened linker were cloned similarly; they are Loop8-ACAD9 and Loop12-ACAD9, in which residues between Thr450 and Asp480 were replaced by 8 and 12 residues of GGS repeats, respectively. cDNAs that code mature proteins of human mitochondrial ECSIT (UniProtKB-Q9BQ95) and NDUFAF1 (UniProtKB-Q9Y375) were purchased from GenScript (Piscataway, USA) as codon-optimized for E. coli expression. Both ECSIT and NDUFAF1 cDNAs were then subcloned into the pET28a vector for expression. Also, the N-terminal domain of ESCIT (Ser49Ser269, which excludes the first 48-residue mitochondrial targeting sequence), hereafter referred to as NECSIT, and the C-terminal domain of ESCIT (Leu249-Ser431), referred to as C-ECSIT, were also cloned into pET28a vector. In order to increase the solubility of the expressed NDUFAF1 protein, the NDUFAF1 cDNA was subcloned into a His6-SMT3-containing pET28a vector and expressed as a SUMO fusion protein (referred to as His $_{6}-$ SMT3-NDUFAF1). The His ${ }_{6}-\mathrm{SMT} 3$ tag was cleaved from NDUFAF1 by protease ULP1 when needed.

Both ACAD9 and ACAD9-His 6 were expressed and purified as described previously $(2,6)$ with some modifications. Both ECSIT and NDUFAF1 proteins were expressed in BL21(DE3) cells and purified in a similar manner as ACAD9-His 6 (6). Briefly, E. coli cells transformed with ECSIT or NDUFAF1 plasmid were grown at $30^{\circ} \mathrm{C}$ until OD reached 0.6 at $600 \mathrm{~nm}$, then induced with $0.5 \mathrm{mM} \mathrm{IPTG}$ at $16^{\circ} \mathrm{C}$ and allowed to grow overnight. All the proteins were purified by Ni-NTA affinity column to SDS-PAGE homogeneity and dialyzed in the HPLC buffer (25mM Tris- $\mathrm{HCl}, \mathrm{pH} 8.0,150 \mathrm{mM} \mathrm{NaCl}$, and $5 \%$ glycerol) before they were further purified by size exclusion chromatography. For each protein, the peak portion corresponding 
to ACAD9 dimer, ECSIT dimer, C-ECSIT tetramer, or His 6 -SMT3-NDUFAF1 monomer was collected and concentrated, and $20 \%$ glycerol was added to each protein solution. Purified proteins were frozen in liquid nitrogen and stored at $-80^{\circ} \mathrm{C}$.

\section{Determination of Protein Concentration.}

Concentrations of all purified proteins were estimated according to their UV absorbance at $280 \mathrm{~nm}$. The apo-protein absorbance extinction coefficient at $280 \mathrm{~nm}$ was calculated according to the online Protein Calculator v3.4 (C. Putnam, The Scripps Research Institute, La Jolla, CA). Calculated molar extinction coefficients at 280nm wavelength of ECSIT, C-ECSIT, and His6-SMT3-NDUFAF1 are $43.8 \mathrm{mM}^{-1} \mathrm{~cm}^{-1}$, $30.4 \mathrm{mM}^{-1} \mathrm{~cm}^{-1}$ and $49.5 \mathrm{mM}^{-1} \mathrm{~cm}^{-1}$, respectively. The calculated molar extinction coefficient for apoACAD9 (not including FAD) is $31.9 \mathrm{mM}^{-1} \mathrm{~cm}^{-1}$. In order to estimate the ACAD9 protein concentration and its FAD content, FAD molar extinction coefficients of $12 \mathrm{mM}^{-1} \mathrm{~cm}^{-1}$ at $450 \mathrm{~nm}$ and $22 \mathrm{mM}^{-1} \mathrm{~cm}^{-1}$ at $280 \mathrm{~nm}$ were used.

\section{Enzymatic Activity Assay: Measurement of $\alpha-\beta$ dehydrogenation of fatty acyl-CoA (fatty acid oxidation activity)}

The fatty acid oxidation (FAO) activity, i.e., $\alpha-\beta$ dehydrogenation activity of fatty acyl-CoA for ACAD9 or VLCAD was assayed using $50 \mu \mathrm{M}$ palmitoyl-CoA as the substrate and $200 \mu \mathrm{M}$ ferricenium hexafluorophosphate as the final electron acceptor, as described by Lehman et al. (17). To examine the ECSIT effect on the FAO activity of ACAD9, various amounts of ECSIT were added to the ACAD9 protein solution and incubated on ice for 30 minutes prior to the activity assay. 1 unit of the enzyme FAO activity is defined as $1 \mu$ mole of ferrocene produced in one minute per $\mu$ mole ACAD9 or VLCAD monomer.

\section{Ni-NTA Pull-Down Assay}

Two proteins were mixed (about $5 \mu \mathrm{M}$ each final concentration) in $0.5 \mathrm{~mL}$ buffer B (25mM Tris- $\mathrm{HCl} \mathrm{pH}$ $8.0,300 \mathrm{mM} \mathrm{NaCl}$, and $5 \%$ glycerol) and incubated on ice for 30 minutes. Ni-NTA resin $(50 \mu \mathrm{L})$ was then added to the protein mixture and further incubated at $4^{\circ} \mathrm{C}$ for 1 hour while mixing in a cold room using a rotator. The protein-bound Ni-NTA resin was then washed three times with $500 \mu \mathrm{L}$ of $20 \mathrm{mM}$ imidazole before eluting with $100 \mu \mathrm{L}$ of $200 \mathrm{mM}$ imidazole in buffer B. The eluted fractions were directly analyzed by SDS PAGE.

\section{Size Exclusion Chromatography}

An HPLC size-exclusion column (Bio-Rad Enrich SEC 650, 10/30) was run at a flow rate of $0.4 \mathrm{ml} / \mathrm{min}$ using a Shimadzu Prominence HPLC system. For a typical chromatography for purification, about 1-5mg 
protein in $0.5 \mathrm{ml}$ buffer was injected, while in a typical chromatography for analytical characterization, 200ul containing about $50-200 \mu \mathrm{g}$ of protein in running buffer $(25 \mathrm{mM}$ Tris- $\mathrm{HCl}, \mathrm{pH} 8.0,150 \mathrm{mM} \mathrm{NaCl}$, and 5\% glycerol) was injected. The column was calibrated with a set of standard molecular weight markers, cytochrome $c(12.3 \mathrm{kDa})$, ovalbumin $(44 \mathrm{kDa})$, bovine serum albumin $(66 \mathrm{kDa}), \operatorname{IgG}(158 \mathrm{kDa})$, apoferritin $(443 \mathrm{kDa})$, and thyroglobulin $(670 \mathrm{kDa})$.

\section{Small-Angle X-ray Scattering}

All three proteins, ACAD9 $\left(7.1 \mathrm{mg} / \mathrm{ml}\right.$, dimeric protein), ECSIT $\left(0.41 \mathrm{mg} / \mathrm{ml}\right.$, also dimeric), and His $6^{-}$ SMT3-NDUFAF1 $(0.52 \mathrm{mg} / \mathrm{ml}$, monomeric), were purified by HPLC and prepared in $25 \mathrm{mM}$ Tris-HCl pH8.0, 150mM NaCl, 0.1mM DTT, and 5\% glycerol. For the binary complex ACAD9/ECSIT and the ternary complex ACAD9/ECSIT/His ${ }_{6}$-SMT3-NDUFAF1, the corresponding proteins were mixed in an equimolar ratio and concentrated to $\geq 1 \mathrm{mg} / \mathrm{ml}$, immediately prior to the SEC-SAXS experiments. SECSAXS experiments were conducted at the 18-ID Biophysics Collaborative Access Team beam-line (BioCAT), Advanced Photon Source, Argonne National Laboratory (APS-ANL), Chicago, IL (18) during the BioCAT Advanced SAXS Training Course, held in October 2017. All samples were applied to a $24 \mathrm{ml}$ GE Superose 6 Increase column, which was directly coupled to the SAXS cell. SAXS measurements were taken both immediately before and after the protein peaks to establish the baseline scattering. BioCAT Beamline specific pipelines were used for data collection. The ATSAS suite (19) was used for data reduction; PRIMUS (20) for Guinier Analysis; and GNOM (21) was used to calculate the radius of gyration, $\mathrm{Rg}$, and the pair-distance distribution function, $\mathrm{P}(\mathrm{r})$. The low-resolution ab initio models were first calculated in DAMMIF (22) with P2 symmetry. The 20 initial models of ACAD9, 40 initial models of the ACAD9/ECSIT binary complex, and 40 initial models of the ACAD9/ECSIT/His6-SMT3-NDUFAF1 ternary complex were clustered and averaged using DAMCLUST (23). The clustered models were run on DAMSTART (24) and refined by DAMMIN (25) using the DAMSTART beads model pdb file as the initial model. The homology model of ACAD9 was obtained from the SWISS-MODEL server (26). Structures of NDUFAF1 and ECSIT domains were modeled using the Robetta web server (27) and the GalaxyGemini server(28) (see Supporting Information for details). SMT3 structure was derived from its X-ray crystal structure (pdb code: 3PGE). For ACAD9, SUPCOMB (29) was used to superimpose the homologymodeled structures onto the final SAXS ab initio beads model. For the ACAD9/ECSIT binary complex and the ACAD9/ECSIT/His6-SMT3-NDUFAF1 ternary complex, structures of ACAD9, ECSIT, NDUFAF1, and SMT3 domains were manually placed onto their SAXS ab initio beads models using the UCSF Chimera program (30). Finally, the SAXS curves calculated from these homology models were overlaid onto the experimental data using FoXS server (31) (25) 


\section{Results}

\section{Expression and Purification of ACAD9, ECSIT, and NDUFAF1}

Human ACAD9 shares $46.4 \%$ sequence identity and 77.6\% sequence similarity with human VLCAD, suggesting that their structures and biophysical properties are likely very similar. All ACAD9 proteins, including wild type ACAD9 (both ACAD9 and ACAD9-His ${ }_{6}$ ) and their mutant forms, were purified to homogeneity as shown and size exclusion chromatographic (SEC) results. ACAD9 was eluted from a SEC column at the same position as VLCAD (Fig. 1), consistent with their sequence and structural similarity. However, purified wild type ACAD9 proteins (both non-tagged ACAD9 and ACAD9-His 6 ) contained only about 70\% FAD, indicating that ACAD9 has a weaker FAD-binding affinity than does VLCAD (Fig. 1). In addition, the wild type ACAD9 protein has a dehydrogenation activity of 131 units. However, in the presence of 100-fold excess exogenous FAD in the assay mix, the activity was increased to 179 units, suggesting that all ACAD9 molecules (including the apo-ACAD9) are active in the presence of exogenous FAD. However, this activity is only $18 \%$ of VLCAD's activity (995 units) under the same conditions (Table 1). Some of the complex I deficient mutants, Arg469Trp, Arg518His, and Arg532Trp have similar dehydrogenation activity to that of wild type ACAD9 in the presence of excess FAD, while other mutants contain little or no dehydrogenation activity. Interestingly, while the Loop8-ACAD9 mutant is not stable (only inclusion bodies were produced upon expression), Loop12-ACAD9 is entirely stable and contains about the same activity as wild type ACAD9 in the presence of excess FAD (Table 1). As expected, no FAO activity was detected with the catalytic-residue mutant, Glu426Gln. All of these mutants exist as homodimers as determined by SEC. Taken together, there is no direct relationship between the FAO activity and complex 1 assembly ability. In addition, it appears that although the sequence of the linker is not important, but it needs a minimum length of $\sim 12$ residues.

Both wild type ECSIT and its C-terminal domain (C-ECSIT) have been successfully expressed and purified to homogeneity, as shown by SDS-PAGE and further verified by MALDI-TOF mass spectra (Fig. 2). However, the purified wild type ECSIT protein revealed multiple peaks in size exclusion chromatography (SEC), revealing the protein is poly dispersed, i.e., multiple aggregated states. To obtain non-aggregated, mono-dispersed protein, we used a stepwise gradient of imidazole concentration, when eluting the Histagged ECSIT from the Ni-affinity column. The fraction eluted at $80 \mathrm{mM}$ imidazole concentration had the largest amount of the apparent-low molecular-weight protein. This fraction was further fractionated on a size exclusion column. The final product of ECSIT purified from SEC had an apparent molecular weight of $\sim 110 \mathrm{kDa}$, consistent with its dimeric form. The fraction eluted with $120 \mathrm{mM}$ or higher concentration of imidazole contained oligomers of higher molecular weights. Even the purified, dimeric ECSIT protein had 
a tendency to further aggregate at high protein concentrations. Thus, purified ECSIT was concentrated to only $0.1-0.5 \mathrm{mg} / \mathrm{mL}$ in Tris buffer (25 mM Tris $\mathrm{HCl}, \mathrm{pH} 7.5,100 \mathrm{mM} \mathrm{NaCl}$ with $20 \%$ glycerol). Repeated cycles of freezing and thawing did not appear to result in the formation of protein aggregates or precipitation, as assessed by the SEC results. Although expression of the N-terminal domain of ECSIT was not successful, the C-terminal domain of ECSIT could be expressed and purified to homogeneity using the same purification procedure used for the full-length ECSIT. Interestingly, the C-ECSIT exists as a homotetramer based on the SEC results - see Figure 6B. It is more stable than the dimeric full-length ECSIT, judging by the fact that the protein was much more soluble and did not aggregate as easily as the full-length protein. Removing the N-terminal His-tag from ECSIT and C-ECSIT did not improve either protein's stability.

Although wild type His6-NDUFAF1 (using NDUFAF1_pET28a) could be expressed and purified similarly to homogeneity as assessed by SDS-PAGE, the purified His-tagged protein was highly aggregated, and a large portion of the protein was eluted in the void volume on a size exclusion column. However, His $6^{-}$ SMT3-NDUFAF1 was much more stable, and its molecular weight was estimated to be $\sim 48 \mathrm{kDa}$ by SDSPAGE, consistent with its expected value. A size-exclusion chromatogram on a Biorad Enrich SEC 650 column gave a homogenous single peak with an apparent molecular weight around $60 \mathrm{kDa}$, indicating it exists as a monomer in solution (Fig. S1). The higher apparent molecular weight by SEC may suggest that the His6-SMT3-NDUFAF1 is not a compact globular protein. The His 6 -SMT3 fusion tag could be cleaved by the ULP1 enzyme; however, the resulting tag-free NDUFAF1 was highly aggregated, and almost $90 \%$ of NDUFAF1 was precipitated during this process. Therefore, His ${ }_{6}$-SMT3-NDUFAF1 protein was directly used in most subsequent experiments, unless otherwise noted

\section{ECSIT Deflavinates ACAD9 and Abolishes Its Dehydrogenation Activity}

It has been previously demonstrated that ACAD9's ability as a dehydrogenase plays no role in the complex I assembly function (4). However, the effect of ESCIT on the enzyme activity of ACAD9 has not been tested directly. We set out to titrate the enzyme activity of ACAD9 by the addition of varying amounts of ECSIT. Although purified ACAD9 in the absence of ECSIT has dehydrogenation activity, it does not have any enzymatic activity in the presence of an excess amount of ECSIT (Table 1). As shown in Figure 3A, excluding the first $\sim 30 \%$ saturation point, the specific activities declined linearly as the concentration of ECSIT increased. When the ECSIT: ACAD9 ratio reached 1:1, almost all the ACAD9 FAO dehydrogenation activity was lost. The likely reason for the first 30\% ECSIT does not follow this linear relationship is that $\sim 30 \%$ of ACAD9 had no bound-FAD and hence had no enzymatic activity. It also suggested that apo-ACAD9 might have a higher binding affinity toward ECSIT than holo ACAD9 with bound FAD. Figure $3 \mathrm{~b}$ shows that C-ECSIT had the same ability to abolish the ACAD9 FAO activity as 
the full-length ECSIT. Furthermore, the loss of FAO activity in the presence of ECSIT was reversible for both the full-length ECSIT and the C-terminal domain C-ECSIT. Figure 3C shows that the FAO activities of both the binary (HPLC-purified, FAD-free ACAD9-ECSIT) and ternary (ACAD9-ECSIT-NDUFAF1) complexes could be recovered when a large amount of exogenous FAD was added.

Further experiments confirmed that the loss of the ACAD9 dehydrogenase activity in the presence of ECSIT was due to the loss of the bound FAD cofactor of ACAD9. As shown in Figure 4, when ACAD9 was incubated with a two-fold molar excess of ECSIT and dialyzed against buffer overnight, almost all the FAD that was bound to ACAD9 was released, as demonstrated by the disappearance of distinct FAD visible peaks at 380nm and 450nm. In comparison, ECSIT did not affect VLCAD, either on the FAD release or the FAO activity.

It appears that both ECSIT and C-ECSIT bind to ACAD9 with similar affinities. The loss of ACAD9 FAO activity induced by binding to ECSIT or C-ECSIT can be reversed by the addition of a large excess of free FAD. Therefore, it is likely that the C-terminal domain of ECSIT directly binds to ACAD9. Furthermore, both ECSIT and C-ECSIT can de-flavinate ACAD9 mutants, including R469W, R518H, R532W, and Loop12-ACAD9, resulting in inactivation of these mutants' FAO activity (Table 1). Since FAD binds to the N-terminal domain of ACAD9, and the mutation sites of these mutants are all located in the C-terminal domain of ACAD9, it is also most likely that ECSIT interacts with the N-terminal domain of the ACAD9 homodimer.

\section{Pull-Down Assays using Ni-NTA Affinity Chromatography reveal interactions between ACAD9, ECSIT, and NDUFAF1}

To further study the nature of interactions between ACAD9 and ECSIT, we also performed pull-down assays using a Ni-NTA affinity column. Attempts to use His-tagged ECSIT to bind ACAD9 were not successful due to a high background coming from the non-specific binding of the ACAD9 protein onto the Ni-NTA resin. To overcome this, we added a His6-tag at the C-terminus of ACAD9 (ACAD9-His6), which behaved the same as the wild type ACAD9, i.e., it exists as a homodimer and retains similar FAO activity. As shown in Figure 5, lanes 6 and 7, ACAD9-His6 pulled down both ECSIT and C-ECSIT efficiently, almost with a near 1:1 molar ratio, when the full-length ECSIT was used. As a control, His 6 -ECSIT failed to pull down VLCAD, as shown in lane 13. Consistent with the results that FAD of ACAD9 was released, when mixed with ECSIT and dialyzed overnight (Figure 4), both pull-down products contained no FAD judging from their UV-Vis spectra (Figure 6). Full-length His ${ }_{6}$-ECSIT can also pull down NDUFAF1 (lane 9 in Figure 5). However, neither ACAD9 nor C-ECSIT can interact with NDUFAF1 directly. When ACAD9-His 6 was mixed with both tag-free ECSIT and His6-SMT-NDUFAF1, the elution product 
contained all three components (lane 8), indicating that the ternary complex was formed. Like the ACAD9/ECSIT binary complex, this ternary complex also contained no FAD and had no FAO activity. These pull-down results are consistent with a model in which ACAD9 binds to the C-terminal domain of ECSIT, and NDUFAF1 binds to the N-terminal domain of ECSIT.

\section{Molecular Weight Determination of the Binary and Ternary Complexes by Size Exclusion Chromatography}

Figure 6A shows the elution profiles of ACAD9 alone, ECSIT alone, and a 1:1 mixture of ACAD9 and ECSIT on a size exclusion column (Biorad Enrich SEC 650). The ACAD9/ECSIT binary complex was eluted at an apparent MW of about $272 \mathrm{kDa}$ (black), which corresponds to one ACAD9 dimer (green) plus one ECSIT dimer (red). The elution profile of the ACAD9+ECSIT mixture at $450 \mathrm{~nm}$ wavelength also clearly showed that the FAD was released from ACAD9 in the ACAD9+ECSIT mixture and was eluted as free FAD much later than the complex peak (gold peak in Fig. 6A). Similarly, Figure 6B shows the binary complex formation of ACAD9/C-ECSIT and the resulting ACAD9 deflavination. However, the C-terminal domain of ECSIT was eluted at apparent MW of $89 \mathrm{kDa}$ (red), indicating that the C-terminal domain of ECSIT exists as a tetramer, rather than as a dimer form as the full-length form. Consequently, the binary complex of ACAD9/C-ECSIT was eluted at apparent MW of $\sim 375 \mathrm{kDa}$ (black), corresponding to a complex of one C-ECSIT tetramer and two ACAD9 dimers (blue). The released FAD from ACAD9 was also eluted at the free FAD position, confirming again that ACAD9 was deflavinated upon complex formation with CECSIT.

To study interactions between ECSIT and NDUFAF1, we used His $6_{-}$-SMT3-NDUFAF1, rather than untagged NDUFAF1, due to the instability of untagged NDUFAF1. Figure 6C shows that the elution peak of the His 6 -SMT3-NDUFAF1/ECSIT complex is much broader (Fig 6C, black profile), indicating that the binary complex is relatively polydispersed. In addition, the relatively sharp tall peak of excess free NDUFAF1 might imply that a portion of ECSIT existed as higher oligomers rather than dimers during sample concentration and consequently lost its ability to interact with NDUFAF1. Nevertheless, the His6SMT3-NDUFAF1/ECSIT binary complex has an apparent MW of 251kDa based on SEC analysis. This MW is also about 30\% larger than the calculated MW of $197 \mathrm{kDa}$, based on the binary complex of one ECSIT dimer and two His6-SMT3-NDUFAF1 monomers, indicating the non-globular nature of the binary complex.

Figure 6D shows that mixing all three purified, individual proteins forms a ternary complex ACAD9/ECSIT/NDUFAF1 with a 1:1:1 molar ratio. ACAD9, ECSIT, and His6-SMT3-NDUFAF1 were co-eluted from the SEC column at an apparent MW of $623 \mathrm{kDa}$ (black profile). As with the binary 
ACAD9/ECSIT complex, FAD was released from ACAD9 upon the ternary complex formation (gold profile). The large apparent molecular weight of the ternary complex at $623 \mathrm{kDa}$ implies that each complex molecule contains four monomers each of ACAD9, ECSIT, and His6-SMT3-NDUFAF1. Remarkably, the ternary complex was very stable and did not aggregate easily and was stable in the presence of $25 \mathrm{mM} \beta$ octylglucoside. In addition, the fusion protein tag (His6-SMT3) could be safely cleaved from the ternary complex without forming precipitation or aggregation. The ternary complex without the His6-SMT3-tag has the same elution profile as the tagged protein (Figure 7). Furthermore, ACAD9 mutants that contained full FAO activities, including Arg469Trp, Arg518His, and Loop12-ACAD9, formed the ternary complex with ECSIT and NDUFAF1. So did the FAO-inactive, catalytic residue mutant, Glu426Gln. The enzymebound FAD was also released from these variants when they formed a complex with ECSIT and NDUFAF1, as shown in Figure S1.

The fact that Arg469Trp and Arg518His also make the ternary complex with ECSIT and NDUFAF1, and yet they are complex1-deficient mutants suggests that the C-terminal domain of ACAD9, which does not interact with ECSIT nor NDUFAF1, must interact with another assembly factor, such as TMEM126B (32) or interact with a complex 1 subunit.

\section{SEC-SAXS Studies of ACAD9, ACAD9/ECSIT (binary complex), and ACAD9/ECSIT/NDUFAF1 (ternary complex)}

Small Angle X-ray Scattering (SAXS) has emerged as a very powerful technique for the study of flexible and less compact roteins, providing important information including the overall size and 1 shape of macromolecules in solution. Figure S2 displays the averaged and normalized experimental SEC-SAXS scattering curves and their P(r) fittings (pair-distance distribution) of the ACAD9 dimer alone (Fig. S2a), ACAD9/ECSIT binary complex (Fig. S2b), and ACAD9/ECSIT/His ${ }_{6}$-SMT3-NDUFAF1 ternary complex (Fig. S2c). The overall parameters obtained from the scattering data are presented in Table 2. The molecular weights of ACAD9 alone and its binary and ternary complexes are in line with their molecular weights estimated by size exclusion chromatography and calculated according to their amino acid sequences.

\section{Homology modeling of the ternary complex, ACAD9/ECSIT/NDUFAF1}

No experimentally determined structure of ACAD9, ECSIT, or NDUFAF1 is available to date. However, ACAD9 is believed to have a very similar structure to that of VLCAD $(4,33)$, and the results of our biophysical characterization (SEC and SAXS data) are consistent with this supposition. Figure 8 shows a cartoon drawing of the human ACAD9 structure modeled after the VLCAD crystal structure ((34), pdb 
Code: 3B96), showing the sites of known complex I deficient variants. However, almost no structural information is currently available for either NDUFAF1 or ECSIT.

For these two proteins, therefore, we used the web-based Robetta programs (27) for domain prediction and model construction. The C-terminal domain of NDUFAF1 (from Leu117 to Lys327) was constructed based on its homology of the galactose-binding domain-like protein, exo- $\beta$-agarase Aga50D protein of marine bacterium Saccharophagus degradans (pdb code: 4BQ5), with a confidence score of 0.643 . The five homology models calculated from Robetta aligned well except for the last twenty residues (Figure S3). However, the N-terminal domain of NDUFAF1 (from Tyr25 to Leu117) has no good homology model, and Robetta gave only a 0.208 confidence score with an uncharacterized protein (pdb code: $3 \mathrm{H} 36$ ). Therefore, the five models were calculated by the de novo method (Figure S3, only first model shown).

For the ECSIT structure, we used two web-based program packages, the Robetta program package (27) and the Phyre2 package (35). The C-terminal domain of ECSIT from Ile267 to Ser431 (the purified C-terminal domain protein, C-ECSIT, consists of Ser249 to Ser431) was further divided into two domains according to the Robetta domain prediction. Of these three domains of ECSIT, the structure of the N-terminal domain (from Ser49 to Gly266, excluding the 48-residue mitochondrial targeting signal) was calculated from the structure of pentatricopeptide repeat protein (pdb code: 4OE1) with a confidence score of 0.249. Although this domain contained only helices, the overall structure based on the five models is relatively flexible (Figure S3). The ECSIT C-terminal domain (from Try341 to Ser431) was predicted to have an isomerase fold (pdb code: 4R3U) with a homology confidence score of 0.282. All five calculated models contained two helices and four beta strands with slightly different conformations (Figure S3). On the other hand, the ECSIT middle domain (Ile267-Gly340) had no good homology model, giving a 0.211 confidence score with a mammalian transport protein (pdb code: 3EGD). However, when this domain was modeled as a homodimer using the GalaxyWeb server (28), it shared a 27\% sequence identity with Bacillus subtilis YojF homodimer protein (pdb code: $1 \mathrm{NJH}$ ). Thus, this domain is most likely responsible for the ECSIT homodimer formation. These results agree well with those obtained for the N-terminal domain using the Phyre2 online server (14). However, Phyre2 was not able to find a good homology model for the C-terminal domain (from Ile267 to Ser431) and predicted it to be distantly related to the pleckstrin homology domain. In addition, the Phyre2 server was not able to predict the dimer formation of ECSIT by its middle domain. Figure S3 shows the overall domain prediction and the structural models obtained by Robetta.

Once the structures of all individual domains were modeled, the docked models between the ECSIT Nterminal domain and the NDUFAF1 C-terminal domain and between the ECSIT C-terminal domain and the ACAD9 dimer were obtained using the ClusPro online server (36-38). These partial models were then 
manually placed onto the corresponding DAMMIN-refined ab initio models using the CHIMERA program (30). Figures S4, S5, and S6 show the possible docking structures of ACAD9 alone, the ACAD9/ECSIT binary complex, and the ACAD9/ECSIT/His6-SMT3-NDUFAF1 ternary complex, respectively, fitting onto the beads models derived from the SAXS data. It should be noted that the ternary complex contains two heterohexamers, and each heterohexamer comprises one ACAD9 dimer, one ECSIT dimer, and two NDUFAF1 monomers. A cartoon model of the ternary heterohexamer complex is shown in Figure 9. The two heterohexamers interact with each other through ECSIT and NDUFAF1 (Figure S6) in the model from our SAXS experiments. Figure S7 shows the calculated SAXS curve based on these models fitting to the SEC-SAXS experimental data.

\section{Discussion}

ACAD9 was first identified by random sequencing as a member of the acyl-CoA dehydrogenase family that catalyzes the $\alpha, \beta$-dehydrogenation reaction of acyl-CoAs, a critical enzymatic activity of the fatty acid $\beta$-oxidation cycle. Like VLCAD, ACAD9 is involved in $\beta$-oxidation of long-chain fatty acids, with optimum chain-length specificity for 16 carbons or longer, consistent with their high-sequence homology $(1,2)$. However, recent studies have shown that ACAD9 also plays an essential role in the assembly of the mitochondrial oxidative phosphorylation complex I (CI) and is co-dependent with two other CI assembly factors, NDUFAF1 and ECSIT (4).

We have demonstrated that ACAD9 directly interacts with ECSIT and forms a stable ternary complex with ECSIT and NDUFAF1. Purified recombinant human ACAD9 expressed in E. coli has only about $20 \%$ of the FAO activity of VLCAD, when C16-CoA is used as the substrate in the presence of excess exogenous FAD. However, when ACAD9 forms a complex with ECSIT, the enzyme-bound cofactor FAD is released from ACAD9, and the enzyme completely loses its FAO activity. Although excess exogenous FAD can restore ACAD9's FAO activity, it is unlikely to act as an FAO dehydrogenase in the presence of ECSIT and NDUFAF1 due to the absence or low concentration of free FAD in the mitochondrial matrix. Therefore, the two roles of ACAD9 as an FAO dehydrogenase and as a chaperone of the C1 assembly factor are not compatible. On the other hand, Heide et al. observed that in rat heart mitochondria, ACAD9 exists by itself (i.e., uncomplexed free ACAD9 molecule), in addition to forming a complex with ECSIT and NDUFAF1 (32). Our results show that ACAD9 can form a binary complex with ECSIT as well as a very stable ternary complex with ECSIT and NDUFAF1. The ternary complex did not break up in the presence of $25 \mathrm{mM}$ OBG detergent. Therefore it is unlikely that the ACAD9 dimer observed in Heide's study (32) is from the dissociation product of the ternary ACAD9/ECSIT/NDUFAF1 complex. Instead, the ACAD9 was likely produced in excess compared to ECSIT in rat heart mitochondria. This is a reasonable assumption because if all ACAD9 is consumed for complex I assembly together with ECSIT 
and NDUFAF1, there would be no FAO activity from ACAD9 at all. Therefore, it appears that ACAD9 plays a dual role as a FAO dehydrogenase and a CI assembly factor, especially in certain organs such as in the brain where ACAD9 is more abundant (2).

\section{ACAD9 vs. VLCAD}

What is the mechanism by which ACAD9 participates in CI assembly? In the current paper, we have characterized the biochemical and biophysical properties of ACAD9 alone, the ACAD9/ECSIT binary complex, and the ACAD9/ECSIT/NDUFAF1 ternary complex. ACAD9 shares $47 \%$ sequence identity and $77 \%$ similarity to VLCAD, suggesting they should have very similar overall structures. In our current study, ACAD9 and VLCAD were eluted in a gel-filtration column at the same time point, corresponding to their homodimer positions. Furthermore, the ACAD9 homology model based on the VLCAD structure (pdb code: 3B96, (34) fits well with our SAXS data (Figure S7A). All these experimental data validate our homology-modeled structure of ACAD9.

However, ACAD9 has about $10 \%$ of the VLCAD's FAO activity. On the other hand, ACAD9 is a C1 assembly factor, while VLCAD is not involved in the $\mathrm{C} 1$ assembly. What are the differences between the two ACADs that make each protein unique? VLCAD is a homodimer and each monomer contains an MCAD-like N-terminal domain and a C-terminal domain linked by a highly mobile arginine-rich region. We have previously reported that not only do both VLCAD and ACAD9 have FAO activity toward long chain acyl-CoAs (C16 or longer), but their trypsin digestion patterns are also very similar (6). In the presence of trypsin, the arginine-rich region between the $\mathrm{N}$-terminal and C-terminal domains was easily cleaved. Both the N-terminal and C-terminal domains were relatively stable, and yet they were still bound to each other after limited trypsin digestion. Thus $90 \%$ of the FAO activity was retained (6).

ACAD9 also behaves very differently from VLCAD. First, VLCAD is much more stable than ACAD9. Although VLCAD can be expressed well as a homodimer with an N-terminal His-tagged protein, ACAD9 is expressed mostly as inclusion bodies under the same conditions. Second, unlike VLCAD, which contains the full stoichiometric amount of FAD, purified wild type ACAD9 with or without the C-terminal His-tag contains at best only about $70 \%$ FAD. At low protein concentrations (less than $20 \mu \mathrm{M}$ ), the bound FAD is further lost to as low as $25 \%$ during gel filtration. Thus, ACAD9 has a lower binding affinity for FAD than does VLCAD. This lower affinity for FAD is not obvious from casual inspection of the two structures. Careful examination of the FAD-binding environment reveals that almost all the residues that directly interact with FAD in ACAD9 and VLCAD are identical. The ribityl-isoalloxazine ring portion of the FAD molecule binds to one monomer of the ACAD9 homodimer, while the ADP moiety of FAD binds to the other monomer near the homodimer interface. Therefore, the lower FAD-binding affinity of ACAD9 is 
most likely due to the higher flexibility of the homodimer interface. Upon comparing their interface interactions, Asp391 in VLCAD, which forms important salt bridges with Arg416 and Arg419 of the other VLCAD monomer, is replaced by Ser395 in ACAD9, while Arg419 of VLCAD is replaced by Leu423 in ACAD9. These residues reside at the center of the helix from residues Y412-I423 at the ACAD9 dimer interface, making the ACAD9 dimer less stable. Several CI-deficient mutants, including Glu413Lys, Arg414Cys, Arg417Cys, and Asp418Gly, also reside on this helix. These mutations severely reduce ACAD9's dimer stability. On the other hand, according to the docking results of ACAD9 and C-ECSIT using Cluspro (38,39), 16 of the 22 final models show C-ECSIT bound to the ACAD9 surface near the FAD pyrophosphate binding site (Figure S8), indicating that the C-terminal domain of ECSIT might be directly responsible for the FAD depletion of ACAD9. These results are entirely consistent with our experimental results. Interestingly, when ClusPro is used to dock the ECSIT C-terminal domain with VLCAD, none of the 30 final models are bound in the same region, i.e., near the PPi moiety of FAD. In fact, they are scattered over a wide range of the molecule. Upon comparing the ECSIT-binding region of ACAD9 and the corresponding region of VLCAD, ACAD9 has more positively charged residues than VLCAD, including R85 (A80, mature VLCAD residue number in parenthesis), R195 (A191), K258 (P255), R317 (G314), K330 (T327), K334 (E331), R335 (K332), R408 (K404), and R609 (G605). Some of these positively charged residues may directly interact with the highly negatively charged ECSIT C-terminal domain. Figure S9 shows a comparison of the electrostatic maps of ACAD9 and VLCAD. ECSIT as a whole is negatively charged with a $\mathrm{pI}$ value of 5.5, but the negative charges are more concentrated on the C-terminal domain (pI of the N-terminal domain from Ser49Gly266 is 9.4, while that of C-terminal domain from Trp341 to Ser431 is 4.1). Since the mutations on the "bottom" of the ACAD9 C-terminal domainArg469Trp, Arg518His, and especially the loop deletion mutant (Loop12-ACAD9) - do not affect ACAD9's interaction with ECSIT, the ACAD9 C-terminal domain is unlikely to interact with ECSIT.

\section{ACAD9 mutation sites}

ACAD9 mutations account for a large number of patients with complex I deficiency. So far, 42 ACAD9 mutations have been found in complex I deficient patients (4). As shown in Figure 8, the mutation sites are almost evenly spread over the ACAD9 molecule. These mutation sites can be divided into three categories according to their locations in the 3D structure: (1) those residues located on the surface of the N-terminal domain that might be involved in interactions with ECSIT (blue balls, e.g., L98S and A220V), (2) residues in the N-terminal domain that might be involved in ACAD9 stability, including those directly involved in the dimer interface interactions (red balls, such as R532W and D418G); and (3) residues in the C-terminal domain that might be involved in interactions with other CI subunits or other assembly factor, e.g., TMEM126B (yellow balls, such as R518H and R469W). 
Providing riboflavin in patients' diets can improve some of these complex I deficient patients caused by ACAD9 mutations, such as S602F, R532W, R518H, D418G, and R414C, but has no effect on other CI deficient patients caused by ACAD9 mutations such as L98S and A220V (40). Since no FAD is needed in the ACAD9/ECSIT/NDUFAF1 complex for CI assembly, it is not well understood why flavin therapy would work for some patients suffering from CI deficiencies caused by ACAD9 mutations. As mentioned earlier, the binding of FAD to apo-ACAD9 enhances the ACAD9 dimer stability. Therefore, it is possible that flavin therapy would improve the stable ACAD9 protein levels in patients with complex 1 deficiency, especially for those mutations that destabilize the ACAD9 dimer interactions. However, since the N module of complex I also contains FMN, it is also possible that flavin therapy could affect complex I activity by stabilizing the $\mathrm{N}$ module of complex I. More studies are needed to determine the effectiveness and mechanism of flavin therapy on patients with ACAD9 mutations.

In HEK293 cells, when ECSIT is knocked down, the protein level of NDUFAF1 is greatly decreased, while NDUFAF1 knockdown results in only a minor decrease in the amount of ECSIT $(4,8,41)$. These results are consistent with our data and correlate well with our ternary complex model. ECSIT is more stable than NDUFAF1, especially when ECSIT is complexed with ACAD9. Although there are no direct interactions between ACAD9 and NDUFAF1, ACAD9 knockdown also greatly decreases the NDUFAF1 protein level (4), since ACAD9 knockdown would affect the ECSIT stability and consequently the NDUFAF1 stability.

\section{The role of ACAD9/ECSIT/NDUFAF1 in CI assembly}

Mammalian complex I consists of 45 subunits, which must be assembled correctly to form a properly functioning mature complex $(42,43))$. The stepwise assembly process includes the formation of many complex I subassemblies or intermediates before they are further assembled into the final mature complex $(12,32,44)$. Many assembly factors, which are not part of the final mature complex I, are often found in complex I intermediates, revealing their functions in CI assembly and stability (32). Among these complex I intermediates, two bands at $\sim 460 \mathrm{kDa}$ and $\sim 830 \mathrm{kDa}$ in blue-native PAGE are well-recognized complex I intermediates containing assembly factors, including ACAD9, ECSIT, and NDUFAF1 $(4,8,41,44)$. Among patients suffering from complex I deficiency (i.e., those who have mutations in both alleles of the NDUFAF1 gene and showed markedly reduced levels of NDUFAF1), the $\sim 460-\mathrm{kDa}$ CI intermediate was degraded to $\sim 400 \mathrm{kDa}$, and the $\sim 830-\mathrm{kDa}$ intermediate and the CI mature enzyme were not formed (41). It has been shown that the ECSIT knockdown greatly decreases the amount of the 500-850kDa intermediate and results in severely impaired complex I assembly (8). ACAD9 knockdown leads to reduced levels of CI, ECSIT, and NDUFAF1 (4). Moreover, pathogenic mutations in ACAD9 that caused isolated CI deficiency in two patients also caused a decrease in the fully assembled CI, as well as in the ECSIT and NDUFAF1 
protein levels (4). The blue-native PAGE also showed an accumulation of the characteristic complex I intermediate at $\sim 500-850 \mathrm{kDa}$. Of the two patients with ACAD9 deficiency, one is of particular interest. The patients has a mutation of $\mathrm{R} 518 \mathrm{H}$ in both alleles of the ACAD9 gene. In our studies, the R518H protein is relatively stable and can form a stable ternary complex with ECSIT and NDUFAF1 (Table 1 and Figure S1). R518 in ACAD9 must be a site that interact with another assembly factor (such as TMEM126B) or a subunit of $\mathrm{C} 1$ molecule.

More recently, Guerrero-Castillo et al. used a dynamic complexome profiling approach to delineate the step-wise formation of these complex I subassemblies and their incorporation into the complete complex I, and gave a more detailed picture of how complex I is assembled (45). Among those profiled intermediates, two correlate well with the characteristic complex I intermediates of $\sim 460 \mathrm{kDa}$ and $\sim 830 \mathrm{kDa}$. One is the intermediate named $\mathrm{P}_{\mathrm{p}}-\mathrm{b}$ with a calculated $\mathrm{MW}$ of $386 \mathrm{kDa}$, which migrates at $\sim 436 \mathrm{kDa}$ in blue-native PAGE and contains subunits ND2, ND3, ND4L, ND6, NDUFC1, and NDUFC2, and assembly factors ACAD9, ECSIT, NDUFAF1, COA1, TMEM186, and TMEM126B. Another is named $\mathrm{Q} / \mathrm{P}_{\mathrm{p}}$ with a calculated MW of $736 \mathrm{kDa}$, which migrates in blue-native PAGE at $\sim 800 \mathrm{kDa}$ and is formed when the $\mathrm{P}_{\mathrm{p}}-\mathrm{b}$ intermediate is integrated with another intermediate named $\mathrm{Q} / \mathrm{P}_{\mathrm{p}}$-a with a calculated $\mathrm{MW}$ of $283 \mathrm{kDa}$. This intermediate migrates at $\sim 298 \mathrm{kDa}$ and contains subunits ND1, NDUFA3, NDUFA8, NDUFA13, NDUFA5, NDUFS2, NDUFS3, NDUFS7, and NDUFS8, and assembly factors NDUFAF4, NDUFAF3, and TIMMDC1 (see Figure 5 of (45)). It should be mentioned that the above calculated MWs of $386 \mathrm{kDa}$ and $736 \mathrm{kDa}$ account for only one ECSIT, one NDUFAF1, and two ACAD9 monomers (one ACAD9 dimer). According to our model, the correct calculated MWs should be $465 \mathrm{kDa}$ and $815 \mathrm{kDa}$, respectively, since there are two ECSIT molecules, two NDUFAF1 molecules, and two ACAD9 monomers in the ACAD9/ECSIT/NDUFAF1 complex. It is likely that, in the $465 \mathrm{kDa} \mathrm{P}_{\mathrm{p}}-\mathrm{b}$ intermediate, NDUFAF1 is responsible for interacting with intermediate $\mathrm{Q} / \mathrm{P}_{\mathrm{p}}-\mathrm{a}(283 \mathrm{kDa})$ to form the $815 \mathrm{kDa} \mathrm{Q} / \mathrm{P}_{\mathrm{b}}$ intermediate. Therefore, knockdown of NDUFAF1 will diminish the $830 \mathrm{kDa} \mathrm{Q} / \mathrm{Pb}$ intermediate, and the $460 \mathrm{kDa}$ band will shift to $390 \mathrm{kDa}$ (without NDUFAF1 in the intermediate). Since ECSIT is responsible for bringing NDUFAF1 and ACAD9 together, the ECSIT knockdown completely disrupts these intermediates. ACAD9 in these intermediates forms higher MW intermediates and is likely responsible for interacting with the CI subunits in the $\mathrm{P}_{\mathrm{D}}$ module. For ACAD9, this interaction is most likely located on the C-terminal domain of ACAD9, while the N-terminal domain of ACAD9 is responsible for interacting with ECSIT. These facts are consistent with the results of our studies of the ACAD9/R518H mutant. The R518H protein forms a stable ACAD9/ECSIT/NDUFAF1 ternary complex in solution and also forms the $500-850 \mathrm{kDa}$ intermediates in cells. However, the mutation in the C-terminal domain impairs the mutant's interactions 
with the $\mathrm{C} 1$ subunits in the $\mathrm{P}_{\mathrm{D}}$ module. These intermediates that contain the $\mathrm{R} 518 \mathrm{H}$ mutation cannot proceed any further in the $\mathrm{C} 1$ assembly and therefore accumulate.

In our current study, we have expressed, purified, and characterized human ACAD9, NDUFAF1, and ECSIT individually and studied the interactions between ACAD9, ECSIT, and NDUFAF1. Figure 9 shows a simplified diagram of our proposed model of the ternary complex ACAD9-ECSIT-NDUFAF1. In this model, ECSIT bridges between NDUFAF1 and ACAD9 through its $\mathrm{N}$ - and C-terminal domains, respectively, while NDUFAF1 and ACAD9 may interact with different complex I subunits and/or other assembly factor(s) during the complex I assembly process. Interestingly, both NDUFAF1 $(41,46)$ and ACAD9 have multiple complex I deficient mutants (Figure 8), while mutants of ECSIT causing C1 deficiency have not been identified so far. Although in vitro, the ACAD9-ECSIT-NDUFAF1 ternary complex exists as a dimer of heterohexamers (dimer ACAD9 + dimer ECSIT + 2 x (NDUFAF1), see Figure S6), in vivo, the ternary complex most likely exists as one heterohexamer, with NDUFAF1 always interacting with other assembly factor (e.g., TMEM126B) or other complex I subunit(s). Both NDUFAF1 alone and the N-terminal domain of ECSIT alone are not stable. When both proteins are expressed alone, both proteins either form high molecular weight aggregates (NDUFAF1) or inclusion bodies (N-ECSIT). Although C-ESCIT can be purified as a relatively stable tetramer, the full-length ECSIT protein is a dimer that aggregates easily, especially at a high concentration $(\geq 1 \mathrm{mg} / \mathrm{ml})$. This is most likely due to the instability of ECSIT's N-terminal domain. When NDUFAF1 is complexed with ECSIT, they stabilize each other through the interactions between NDUFAF1 and the N-terminal domain of ECSIT. This is consistent with the previous observation that ECSIT is required for the stabilization of NDUFAF1 (8).

1. Zhang, J., Zhang, W., Zou, D., Chen, G., Wan, T., Zhang, M., and Cao, X. (2002) Cloning and functional characterization of ACAD-9, a novel member of human acyl-CoA dehydrogenase family. Biochem Biophys Res Commun 297, 1033-1042

2. Ensenauer, R., He, M., Willard, J. M., Goetzman, E. S., Corydon, T. J., Vandahl, B. B., Mohsen, A. W., Isaya, G., and Vockley, J. (2005) Human acyl-CoA dehydrogenase-9 plays a novel role in the mitochondrial beta-oxidation of unsaturated fatty acids. J Biol Chem 280, 32309-32316

3. He, M., Rutledge, S. L., Kelly, D. R., Palmer, C. A., Murdoch, G., Majumder, N., Nicholls, R. D., Pei, Z., Watkins, P. A., and Vockley, J. (2007) A new genetic disorder in mitochondrial fatty acid beta-oxidation: ACAD9 deficiency. Am J Hum Genet 81, 87-103

4. Nouws, J., Nijtmans, L., Houten, S. M., van den Brand, M., Huynen, M., Venselaar, H., Hoefs, S., Gloerich, J., Kronick, J., Hutchin, T., Willems, P., Rodenburg, R., Wanders, R., van den Heuvel, L., Smeitink, J., and Vogel, R. O. (2010) Acyl-CoA dehydrogenase 9 is required for the biogenesis of oxidative phosphorylation complex I. Cell Metab 12, 283-294

5. Nouws, J., Te Brinke, H., Nijtmans, L. G., and Houten, S. M. (2014) ACAD9, a complex I assembly factor with a moonlighting function in fatty acid oxidation deficiencies. Hum Mol Genet 23, 13111319 
6. Schiff, M., Haberberger, B., Xia, C., Mohsen, A. W., Goetzman, E. S., Wang, Y., Uppala, R., Zhang, Y., Karunanidhi, A., Prabhu, D., Alharbi, H., Prochownik, E. V., Haack, T., Haberle, J., Munnich, A., Rotig, A., Taylor, R. W., Nicholls, R. D., Kim, J. J., Prokisch, H., and Vockley, J. (2015) Complex I assembly function and fatty acid oxidation enzyme activity of ACAD9 both contribute to disease severity in ACAD9 deficiency. Hum Mol Genet 24, 3238-3247

7. Kopp, E., Medzhitov, R., Carothers, J., Xiao, C., Douglas, I., Janeway, C. A., and Ghosh, S. (1999) ECSIT is an evolutionarily conserved intermediate in the Toll/IL-1 signal transduction pathway. Genes Dev 13, 2059-2071

8. Vogel, R. O., Janssen, R. J., van den Brand, M. A., Dieteren, C. E., Verkaart, S., Koopman, W. J., Willems, P. H., Pluk, W., van den Heuvel, L. P., Smeitink, J. A., and Nijtmans, L. G. (2007) Cytosolic signaling protein Ecsit also localizes to mitochondria where it interacts with chaperone NDUFAF1 and functions in complex I assembly. Genes Dev 21, 615-624

9. Shahul Hameed, U. F., Sanislav, O., Lay, S. T., Annesley, S. J., Jobichen, C., Fisher, P. R., Swaminathan, K., and Arold, S. T. (2018) Proteobacterial Origin of Protein Arginine Methylation and Regulation of Complex I Assembly by MidA. Cell Rep 24, 1996-2004

10. Zickermann, V., Wirth, C., Nasiri, H., Siegmund, K., Schwalbe, H., Hunte, C., and Brandt, U. (2015) Structural biology. Mechanistic insight from the crystal structure of mitochondrial complex I. Science 347, 44-49

11. Koopman, W. J., Willems, P. H., and Smeitink, J. A. (2012) Monogenic mitochondrial disorders. N Engl J Med 366, 1132-1141

12. Mimaki, M., Wang, X., McKenzie, M., Thorburn, D. R., and Ryan, M. T. (2012) Understanding mitochondrial complex I assembly in health and disease. Biochim Biophys Acta 1817, 851-862

13. Sanchez-Caballero, L., Guerrero-Castillo, S., and Nijtmans, L. (2016) Unraveling the complexity of mitochondrial complex I assembly: A dynamic process. Biochim Biophys Acta 1857, 980-990

14. Giachin, G., Bouverot, R., Acajjaoui, S., Pantalone, S., and Soler-Lopez, M. (2016) Dynamics of Human Mitochondrial Complex I Assembly: Implications for Neurodegenerative Diseases. Front Mol Biosci 3, 43

15. Elurbe, D. M., and Huynen, M. A. (2016) The origin of the supernumerary subunits and assembly factors of complex I: A treasure trove of pathway evolution. Biochim Biophys Acta 1857, 971-979

16. Formosa, L. E., Dibley, M. G., Stroud, D. A., and Ryan, M. T. (2018) Building a complex complex: Assembly of mitochondrial respiratory chain complex I. Semin Cell Dev Biol 76, 154-162

17. Lehman, T. C., Hale, D. E., Bhala, A., and Thorpe, C. (1990) An acyl-coenzyme A dehydrogenase assay utilizing the ferricenium ion. Anal Biochem 186, 280-284

18. Fischetti, R., Stepanov, S., Rosenbaum, G., Barrea, R., Black, E., Gore, D., Heurich, R., Kondrashkina, E., Kropf, A. J., Wang, S., Zhang, K., Irving, T. C., and Bunker, G. B. (2004) The BioCAT undulator beamline 18ID: a facility for biological non-crystalline diffraction and X-ray absorption spectroscopy at the Advanced Photon Source. J Synchrotron Radiat 11, 399-405

19. Franke, D., Petoukhov, M. V., Konarev, P. V., Panjkovich, A., Tuukkanen, A., Mertens, H. D. T., Kikhney, A. G., Hajizadeh, N. R., Franklin, J. M., Jeffries, C. M., and Svergun, D. I. (2017) ATSAS 2.8: a comprehensive data analysis suite for small-angle scattering from macromolecular solutions. J Appl Crystallogr 50, 1212-1225

20. Konarev, P. V., Volkov, V. V., Sokolova, A. V., Koch, M. H. J., and Svergun, D. I. (2003) PRIMUS: a Windows PC-based system for small-angle scattering data analysis. Journal of Applied Crystallography 36, 1277-1282

21. Svergun, D. (1992) Determination of the regularization parameter in indirect-transform methods using perceptual criteria. Journal of Applied Crystallography 25, 495-503

22. Franke, D., and Svergun, D. I. (2009) DAMMIF, a program for rapid ab-initio shape determination in small-angle scattering. J Appl Crystallogr 42, 342-346

23. Petoukhov, M. V., Franke, D., Shkumatov, A. V., Tria, G., Kikhney, A. G., Gajda, M., Gorba, C., Mertens, H. D., Konarev, P. V., and Svergun, D. I. (2012) New developments in the ATSAS program package for small-angle scattering data analysis. J Appl Crystallogr 45, 342-350 
24. Volkov, V. V., and Svergun, D. I. (2003) Uniqueness of ab initio shape determination in smallangle scattering. Journal of Applied Crystallography 36, 860-864

25. Svergun, D. I. (1999) Restoring low resolution structure of biological macromolecules from solution scattering using simulated annealing. Biophys $J$ 76, 2879-2886

26. Waterhouse, A., Bertoni, M., Bienert, S., Studer, G., Tauriello, G., Gumienny, R., Heer, F. T., de Beer, T. A. P., Rempfer, C., Bordoli, L., Lepore, R., and Schwede, T. (2018) SWISS-MODEL: homology modelling of protein structures and complexes. Nucleic Acids Res 46, W296-w303

27. Kim, D. E., Chivian, D., and Baker, D. (2004) Protein structure prediction and analysis using the Robetta server. Nucleic Acids Res 32, W526-531

28. Lee, H., Park, H., Ko, J., and Seok, C. (2013) GalaxyGemini: a web server for protein homooligomer structure prediction based on similarity. Bioinformatics 29, 1078-1080

29. Kozin, M. B., and Svergun, D. I. (2001) Automated matching of high- and low-resolution structural models. Journal of Applied Crystallography 34, 33-41

30. Pettersen, E. F., Goddard, T. D., Huang, C. C., Couch, G. S., Greenblatt, D. M., Meng, E. C., and Ferrin, T. E. (2004) UCSF Chimera--a visualization system for exploratory research and analysis. J Comput Chem 25, 1605-1612

31. Schneidman-Duhovny, D., Hammel, M., Tainer, J. A., and Sali, A. (2013) Accurate SAXS profile computation and its assessment by contrast variation experiments. Biophys $J$ 105, 962-974

32. Heide, H., Bleier, L., Steger, M., Ackermann, J., Drose, S., Schwamb, B., Zornig, M., Reichert, A. S., Koch, I., Wittig, I., and Brandt, U. (2012) Complexome profiling identifies TMEM126B as a component of the mitochondrial complex I assembly complex. Cell Metab 16, 538-549

33. Schiff, M., Mohsen, A. W., Karunanidhi, A., McCracken, E., Yeasted, R., and Vockley, J. (2013) Molecular and cellular pathology of very-long-chain acyl-CoA dehydrogenase deficiency. Mol Genet Metab 109, 21-27

34. McAndrew, R. P., Wang, Y., Mohsen, A. W., He, M., Vockley, J., and Kim, J. J. (2008) Structural basis for substrate fatty acyl chain specificity: crystal structure of human very-long-chain acyl-CoA dehydrogenase. J Biol Chem 283, 9435-9443

35. Kelley, L. A., Mezulis, S., Yates, C. M., Wass, M. N., and Sternberg, M. J. (2015) The Phyre2 web portal for protein modeling, prediction and analysis. Nat Protoc 10, 845-858

36. Kozakov, D., Beglov, D., Bohnuud, T., Mottarella, S. E., Xia, B., Hall, D. R., and Vajda, S. (2013) How good is automated protein docking? Proteins 81, 2159-2166

37. Ignatov, M., Liu, C., Alekseenko, A., Sun, Z., Padhorny, D., Kotelnikov, S., Kazennov, A., Grebenkin, I., Kholodov, Y., Kolosvari, I., Perez, A., Dill, K., and Kozakov, D. (2019) Monte Carlo on the manifold and MD refinement for binding pose prediction of protein-ligand complexes: 2017 D3R Grand Challenge. J Comput Aided Mol Des 33, 119-127

38. Vajda, S., Yueh, C., Beglov, D., Bohnuud, T., Mottarella, S. E., Xia, B., Hall, D. R., and Kozakov, D. (2017) New additions to the ClusPro server motivated by CAPRI. Proteins 85, 435-444

39. Kozakov, D., Hall, D. R., Xia, B., Porter, K. A., Padhorny, D., Yueh, C., Beglov, D., and Vajda, S. (2017) The ClusPro web server for protein-protein docking. Nat Protoc 12, 255-278

40. Repp, B. M., Mastantuono, E., Alston, C. L., Schiff, M., Haack, T. B., Rotig, A., Ardissone, A., Lombes, A., Catarino, C. B., Diodato, D., Schottmann, G., Poulton, J., Burlina, A., Jonckheere, A., Munnich, A., Rolinski, B., Ghezzi, D., Rokicki, D., Wellesley, D., Martinelli, D., Wenhong, D., Lamantea, E., Ostergaard, E., Pronicka, E., Pierre, G., Smeets, H. J. M., Wittig, I., Scurr, I., de Coo, I. F. M., Moroni, I., Smet, J., Mayr, J. A., Dai, L., de Meirleir, L., Schuelke, M., Zeviani, M., Morscher, R. J., McFarland, R., Seneca, S., Klopstock, T., Meitinger, T., Wieland, T., Strom, T. M., Herberg, U., Ahting, U., Sperl, W., Nassogne, M. C., Ling, H., Fang, F., Freisinger, P., Van Coster, R., Strecker, V., Taylor, R. W., Haberle, J., Vockley, J., Prokisch, H., and Wortmann, S. (2018) Clinical, biochemical and genetic spectrum of 70 patients with ACAD9 deficiency: is riboflavin supplementation effective? Orphanet J Rare Dis 13, 120

41. Dunning, C. J., McKenzie, M., Sugiana, C., Lazarou, M., Silke, J., Connelly, A., Fletcher, J. M., Kirby, D. M., Thorburn, D. R., and Ryan, M. T. (2007) Human CIA30 is involved in the early 
assembly of mitochondrial complex I and mutations in its gene cause disease. Embo j 26, 32273237

42. Vinothkumar, K. R., Zhu, J., and Hirst, J. (2014) Architecture of mammalian respiratory complex I. Nature 515, 80-84

43. Wirth, C., Brandt, U., Hunte, C., and Zickermann, V. (2016) Structure and function of mitochondrial complex I. Biochim Biophys Acta 1857, 902-914

44. Formosa, L. E., Muellner-Wong, L., Reljic, B., Sharpe, A. J., Jackson, T. D., Beilharz, T. H., Stojanovski, D., Lazarou, M., Stroud, D. A., Ryan, M. T., Zhu, J., Vinothkumar, K. R., and Hirst, J. (2020) Dissecting the Roles of Mitochondrial Complex I Intermediate Assembly Complex Factors in the Biogenesis of Complex I Structure of mammalian respiratory complex I. Cell Rep 31, 107541

45. Guerrero-Castillo, S., Baertling, F., Kownatzki, D., Wessels, H. J., Arnold, S., Brandt, U., and Nijtmans, L. (2017) The Assembly Pathway of Mitochondrial Respiratory Chain Complex I. Cell Metab 25, 128-139

46. Fassone, E., Taanman, J. W., Hargreaves, I. P., Sebire, N. J., Cleary, M. A., Burch, M., and Rahman, S. (2011) Mutations in the mitochondrial complex I assembly factor NDUFAF1 cause fatal infantile hypertrophic cardiomyopathy. J Med Genet 48, 691-697

\section{Figure Legends}

Figure 1. Overlay of ACAD9 (green) and VLCAD (gold) elution profiles on a size exclusion column (BioRad Enrich SEC 650). The standard molecular weight protein peaks (grey dashed curves) from left to right include thyroglobulin $(670 \mathrm{kDa})$, apoferritin(443kDa), IgG $(158 \mathrm{kDa}), \mathrm{BSA}(67 \mathrm{kDa})$, ovalbumin $(43 \mathrm{kDa})$, and cytochrome $c(12.3 \mathrm{kDa})$.

Figure 2. MALDI-TOF MASS spectra of $\mathrm{His}_{6}$-ECSIT before (A) and after (B) thrombin cleavage of the His-tag, and His ${ }_{6}$-C-ECSIT before (C) and after (D) thrombin cleavage of the His-tag. The calculated MW of His ${ }_{6}$-ECSIT and His ${ }_{6}$-C-ECSIT before and after thrombin cleavage are 46,353, 44,570, 22,960 and 21,177 Da, respectively.

Figure 3. The effect of ECSIT and FAD on ACAD9 remaining FAO activity. The HPLC purified ACAD9 dimer has a specific activity of 131 units. When 100-fold excess exogenous FAD was first incubated with ACAD9 before the assay, the specific activity was increased to 179 units, consistent with the estimate of the FAD content $\sim 70 \%$ by the UV spectral measurement. Therefore, the activity of ACAD9+100X FAD was considered to be $100 \%$ of the wild type ACAD9 specific activity. The remaining ACAD9 FAO activities decrease linearly with the addition of wild type ECSIT (A) or C- 
ECSIT (B). The dashed line shows the remaining ACAD9 activity, which has a linear relationship with the amount of added ECSIT or C-ECSIT. The ratio of ACAD9:ECSIT or ACAD9:C-ECSIT is about 1:1. (C) Exogenous FAD can completely recover ACAD9 FAO activity in ACAD9/ECSIT (red) or ACAD9/ECSIT/NDUFAF1 (black) complex. There is no difference between ACAD9/ECSIT and ACAD9/ECSIT/NDUFAF1 complexes for the FAO activity recovery by excess FAD, indicating that the interactions between ACAD9 and ECSIT is independent of the interactions between ECSIT and NDUFAF1.

Figure 4. Deflavination of ACAD9 but not VLCAD by ECSIT. UV-VIS spectra of VLCAD (red) and ACAD9 (green) when they were mixed with a 1:1 molar ratio with ECSIT and dialyzed overnight. VLCAD $(20 \mu \mathrm{M})$ or ACAD9 $(20 \mu \mathrm{M})$ was mixed with 20uM ECSIT in 100ul Tris buffer $\mathrm{pH} 7.4,100 \mathrm{mM} \mathrm{NaCl}, 10 \%$ glycerol and dialyzed in the same buffer overnight).

Figure 5. Purified ACAD9, ECSIT, and NDUFAF1 pull-down assay on a Ni-NTA affinity column. Lanes 1. MW Marker, 2. ACAD9-His, 3. ECSIT, 4. C-ECSIT, 5. NDUFAF1, 6. Pull-down product of ACAD9-His 6 +ECSIT, 7. Pull-down product of ACAD9-His + + C-ECSIT. 8. Pull-down product of ACAD9-His ${ }_{6}+$ ECSIT+NDUFAF1. 9. Pull-down product of His ${ }_{6}$-ECSIT + NDUFAF1. 10, His -ECSIT, 11. VLCAD, 12. 1:1 mixture of His -ECSIT + VLCAD, 13. Pull-down product of His -ECSIT +VLCAD. Lanes 6, 7, 8 show that ACAD9-His ${ }_{6}$ can pull down either ECSIT, C-ECSIT, or both ECSIT and NDUFAF1 simultaneously. Lane 9 shows His 6 -ECSIT can pull down NDUFAF1. As a control, His ${ }_{6}^{-}$ ECSIT failed to pull down VLCAD shown in lane 13.

Figure 6. Chromatographic elution profiles showing the complex formation of (A) ACAD9/His 6 -ECSIT, (B)ACAD9/His 6 -C-ECSIT, (C) His ${ }_{6}$-ECSIT/His 6 -SMT3-NDUFAF1, and (D) ACAD9/His ${ }_{6}$-ECSIT/His ${ }_{6}^{-}$ SMT3-NDUFAF1. ACAD9, His $_{6}$-ECSIT or His ${ }_{6}$-C-ECSIT, and His ${ }_{6}$-SMT3-NDUFAF1 alone are shown in green, red, and blue, respectively. The black and gold profiles are the mixtures of equimolar individual proteins recorded at $280 \mathrm{~nm}$ and $450 \mathrm{~nm}$, respectively. For better comparison, the absorbance at $450 \mathrm{~nm}$ in gold profiles was multiplied by 6 to 10 times. The standard MW protein peaks(grey) from left to right include thyroglobulin $(670 \mathrm{kDa})$, apoferritin(443kDa), IgG(158kDa), BSA $(67 \mathrm{kDa})$, ovalbumin $(43 \mathrm{kDa})$, and cytochrome $c(12.3 \mathrm{kDa})$. 
Figure 7. Cleavage of the His6-SMT3 tag from His6-SMT3-NDUFAF1 in ACAD9/His6-ECSIT/His6SMT3-ACAD9 complex does not change its stability. A) HPLC elution profile of the 1:1:1 mixture of ACAD9/His6-ECSIT/His6-SMT3-NDUFAF1 is shown in black (the same profile as in Figure 6D), and the tag-free sample is shown in red. The fractions at 26-28 minutes (lane 1 in B) were digested with UPL1 to cleave the His6-SMT3 tag (lane 2 in B) and injected onto the HPLC column. The yellow curve is the HPLC profile at 450nm of the His6-SMT3 tag-cleaved ternary complex (the absorbance value has been scaled up 20 times of the black curve). The small peaks between 45 to 50 minutes in the His6-SMT3 tagfree HPLC profile (red) are from the contaminations rather than FAD due to the small amount of sample injected. The standard MW protein peaks (grey) from left to right include thyroglobulin $(670 \mathrm{kDa})$, apoferritin (443kDa), IgG(158kDa), BSA (67kDa), ovalbumin(43kDa), and cytochrome $c(12.3 \mathrm{kDa})$.

B) SDS-PAGE of the ACAD9/ECSIT/NDUFAF1 ternary complex. Lane 1, the ternary ACAD9/His ${ }_{6}$ ECSIT/His ${ }_{6}$ SMT3-NDUFAF1 complex purified from HPLC (black curve in A). Lane2, protein in lane 1 was digested overnight by UPL1. This digested product was directly injected onto size exclusion column (red curve in A). Lane 3, protein in lane 1 was digested overnight by both UPL1 and thrombin. Lane 4, protein markers from top to bottom band are 70, 60, 50, 40, 30, 25, 20, $15 \mathrm{kDa}$. Band a, b, c, d, e,f are ACAD9, His6-SMT3-NduFAF1, His -ECSIT, NDUFAF1, His -SMT3 tag, and ECSIT, respectively. Double band (X) most likely contains NDUFAF1(lower band) and the product of His6SMT3-NDUFAF1 cleaved by thrombin due to a non-specific cleavage of the SMT3 tag (upper band).

Figure 8. Homology-modeled structure of ACAD9 homodimer with 42 currently known CI deficient mutations marked. The mutations near the surface in the $\mathrm{N}$-terminal domain that might be involved in ECSIT binding are colored blue; the mutations that might decrease the ACAD9 stability including those involved in the dimer interface interactions are colored red; the mutations in the C-terminal domain that are unlikely involved in the dimer interface interactions are colored yellow.

Figure 9. A cartoon model of the ternary complex ACAD9-ECSIT-NDUFAF1 (MCIA complex). The ECSIT C-terminal domain binds to the N-terminal domain of ACAD9 and the N-terminal domain of ECSIT binds to NDUFAF1. 
Table 1. ACAD9 FAO activity in the absence/presence of exogenous FAD or ECSIT

\begin{tabular}{|l|l|l|l|l|l|l|l|l|}
\hline & $\begin{array}{l}\text { ACAD9 } \\
(\text { no tag })\end{array}$ & $\begin{array}{l}\text { ACAD9- } \\
\text { His }_{6}\end{array}$ & $\begin{array}{l}\text { R532W- } \\
\text { His }_{6}\end{array}$ & $\begin{array}{l}\text { R518H- } \\
\text { His }_{6}\end{array}$ & $\begin{array}{l}\text { R469W- } \\
\text { His }_{6}\end{array}$ & $\begin{array}{l}\text { E426Q- } \\
\text { His }_{6}\end{array}$ & $\begin{array}{l}\text { Loop12- } \\
\text { His }_{6}\end{array}$ & $\begin{array}{l}\text { VLCAD } \\
\left(\text { N- } \text { His }_{6}\right.\end{array}$ \\
\hline & $131 \pm 7$ & $109 \pm 7$ & $84 \pm 7$ & $130 \pm 7$ & $122 \pm 6$ & $11 \pm 9$ & $55 \pm 5$ & $985 \pm 75$ \\
\hline +100X FAD & $179 \pm 6$ & $180 \pm 4$ & $181 \pm 9$ & $177 \pm 7$ & $180 \pm 6$ & $11 \pm 7$ & $194 \pm 5$ & $995 \pm 91$ \\
\hline +5X ECSIT & $11 \pm 8$ & $11 \pm 4$ & $15 \pm 4$ & $13 \pm 9$ & $12 \pm 5$ & $10 \pm 2$ & $13 \pm 1$ & $940 \pm 36$ \\
\hline
\end{tabular}

FAO Activity is the average of three measurements in $\mu$ mole (Ferricenium)/minute /umole of ACAD9 monomer

Table 2. Parameters of ACAD9 and its complexes calculated from the SEC-SAXS data.

\begin{tabular}{|l|l|l|l|l|l|l|}
\hline Samples & $\mathrm{R}_{\mathrm{g}}(\mathrm{nm})$ & $\mathrm{D}_{\max }(\mathrm{nm})$ & $\mathrm{V}_{\text {porod }}\left(\mathrm{nm}^{3}\right)$ & $\begin{array}{l}\mathrm{MW}(\mathrm{kDa}) \\
\mathrm{By} \mathrm{SAXS}\end{array}$ & $\begin{array}{l}\text { MW(kDa) } \\
\text { calculated } \\
\text { using } \\
\text { amino acid } \\
\text { sequence }\end{array}$ & $\begin{array}{l}\text { MW(kDa) } \\
\text { estimated } \\
\text { from SEC } \\
\text { data only }\end{array}$ \\
\hline ACAD9 & 3.41 & 10.3 & 230 & 138 & 130 & 113 \\
\hline ACAD9/ECSIT & 5.02 & 16.0 & 494 & 318 & 223 & 272 \\
\hline $\begin{array}{l}\text { ACAD9/ECSIT/His6- } \\
\text { SMT3-NDUFAF1 }\end{array}$ & 6.78 & 21.3 & 1540 & 715 & 637 & 623 \\
\hline
\end{tabular}

The abbreviations used are as follows: Rg, radius of gyration; Dmax, maximum size of the particle; VP, excluded volume of the hydrated particle; MW, experimental molecular mass of the solute. 


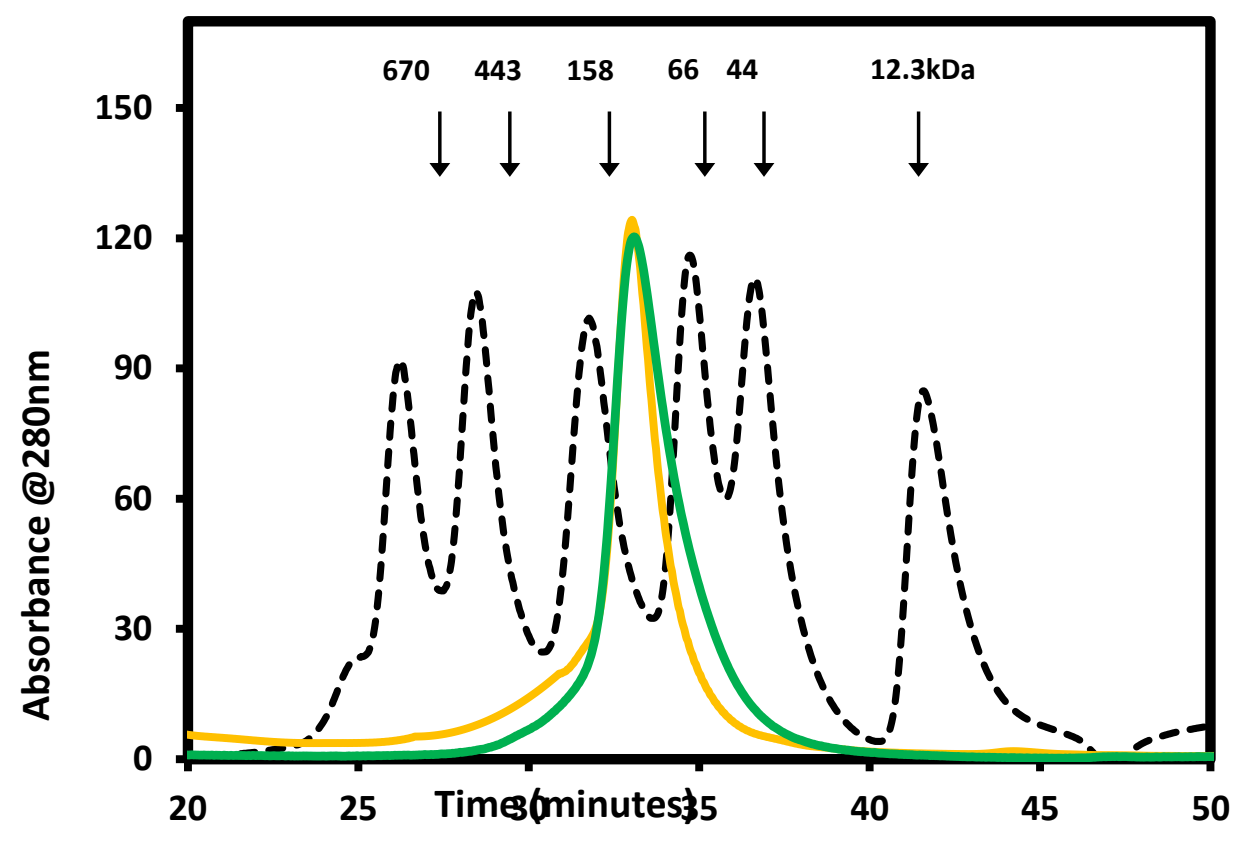

Figure 1. Overlay of ACAD9 (green) and VLCAD (gold) elution profiles on a size exclusion column (BioRad Enrich SEC 650 ). The standard MW protein peaks (grey dashed curves) from left to right include thyroglobulin $(670 \mathrm{kDa})$, apoferritin(443kDa), IgG (158kDa), BSA (67kDa), ovalbumin(43kDa), cytochrome $c(12.3 \mathrm{kDa})$. 


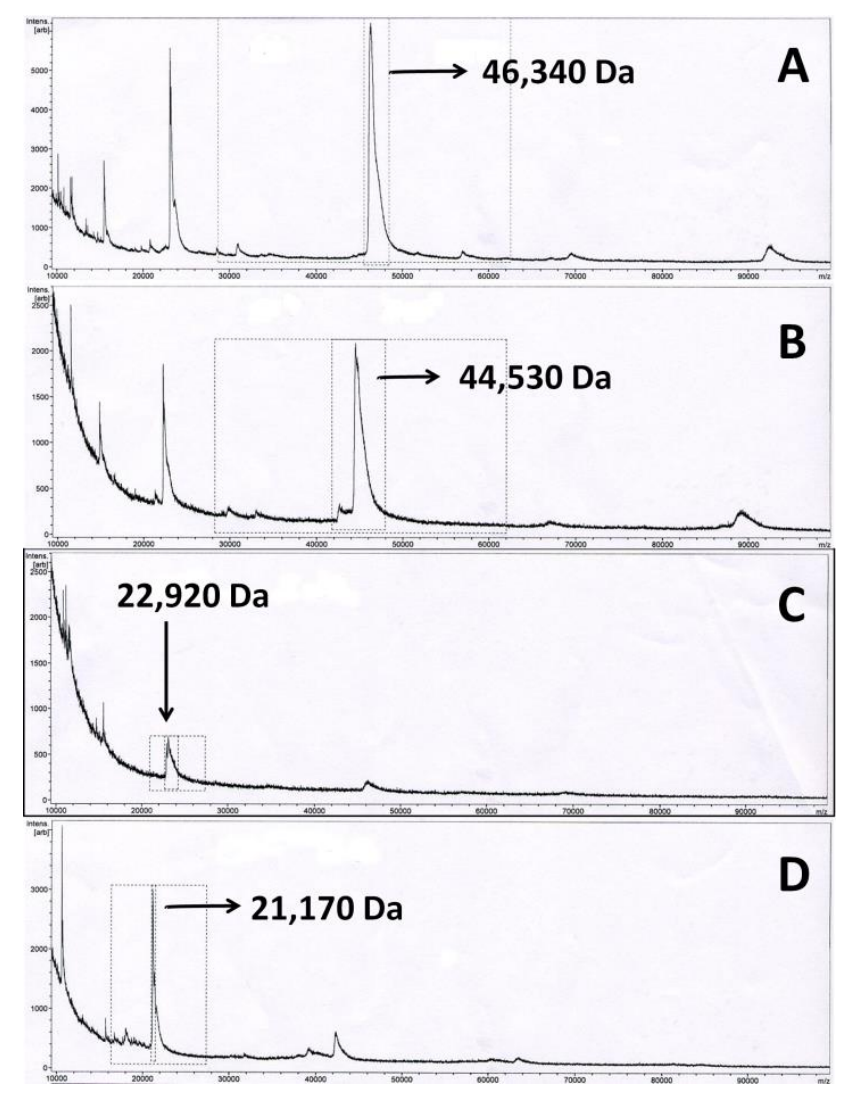

Figure 2. MALDI-TOF MASS spectra of His $_{6}$-ECSIT before (A) and after (B) thrombin cleavage of the His-tag, and $\mathrm{His}_{6}$-C-ECSIT before (C) and after (D) thrombin cleavage of the His-tag. The calculated MW of $\mathrm{His}_{6}$-ECSIT and $\mathrm{His}_{6}-\mathrm{C}-$ ECSIT before and after thrombin cleavages are 46,353, 44,570, 22,960 and 21,177 Da, respectively. 

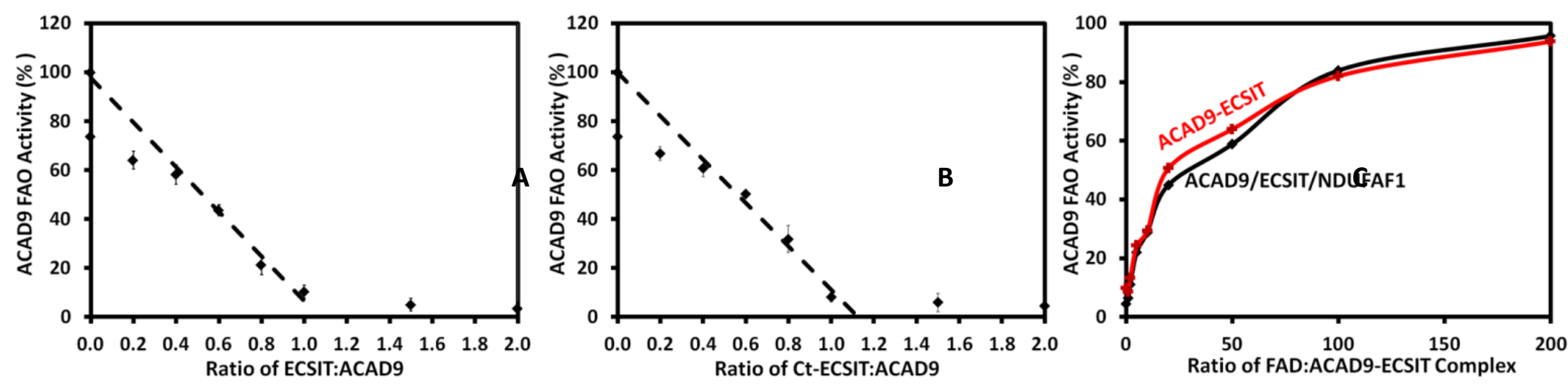

Figure 3. The effect of ECSIT and FAD on ACAD9 remaining FAO activity. The HPLC purified ACAD9 dimer has a specific activity of 131 units. When 100-fold excess exogenous FAD was first incubated with ACAD9 before the assay, the specific activity was increased to 179 units, consistent with the estimate of the FAD content $\sim 70 \%$ by the UV spectral measurement. Therefore, the activity of ACAD9+100X FAD was considered to be $100 \%$ of the wild type ACAD9 specific activity. The remaining ACAD9 FAO activities decrease linearly with the addition of wild type ECSIT (A) or C-ECSIT (B). The dashed line shows the remaining ACAD9 activity, which has a linear relationship with the amount of added ECSIT or C-ECSIT. The ratio of ACAD9:ECSIT or ACAD9:C-ECSIT is about 1:1. (C) Exogenous FAD can completely recover ACAD9 FAO activity in ACAD9/ECSIT (red) or ACAD9/ECSIT/NDUFAF1 (black) complex. There is no difference between ACAD9/ECSIT and ACAD9/ECSIT/NDUFAF1 complexes for the FAO activity recovery by excess FAD, indicating that the interactions between ACAD9 and ECSIT is independent of the interactions between ECSIT and NDUFAF1. 


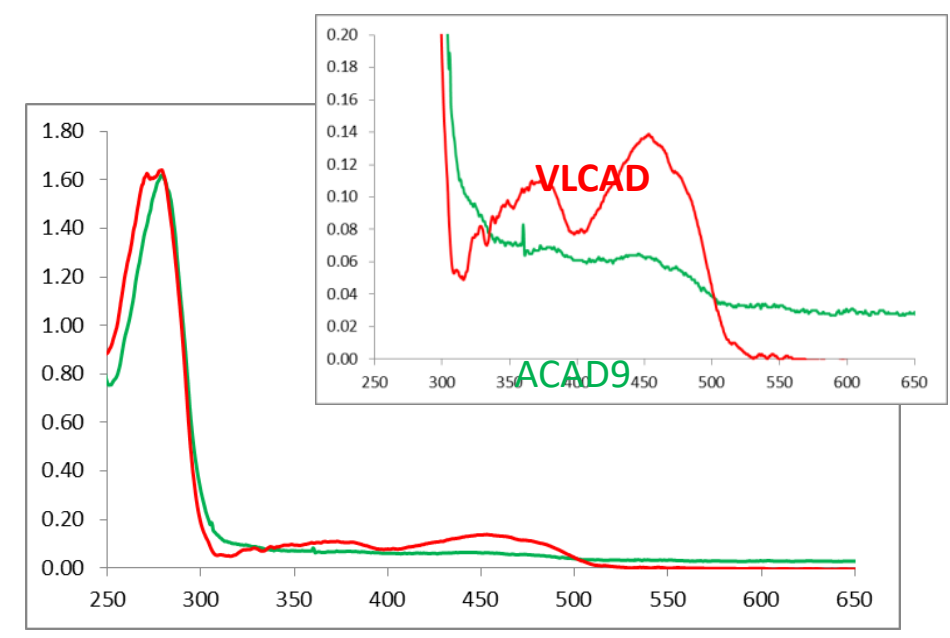

Figure 4. Deflavination of ACAD9 but not VLCAD by ECSIT. UV-VIS spectra of VLCAD (red) and ACAD9 (green) when they were mixed with a 1:1 molar ratio with ECSIT and dialyzed overnight. VLCAD $(20 \mu \mathrm{M})$ or ACAD9 $(20 \mu \mathrm{M})$ was mixed with 20uM ECSIT in 100ul Tris buffer $\mathrm{pH} 7.4,100 \mathrm{mM} \mathrm{NaCl}, 10 \%$ glycerol and dialyzed in the same buffer overnight). 


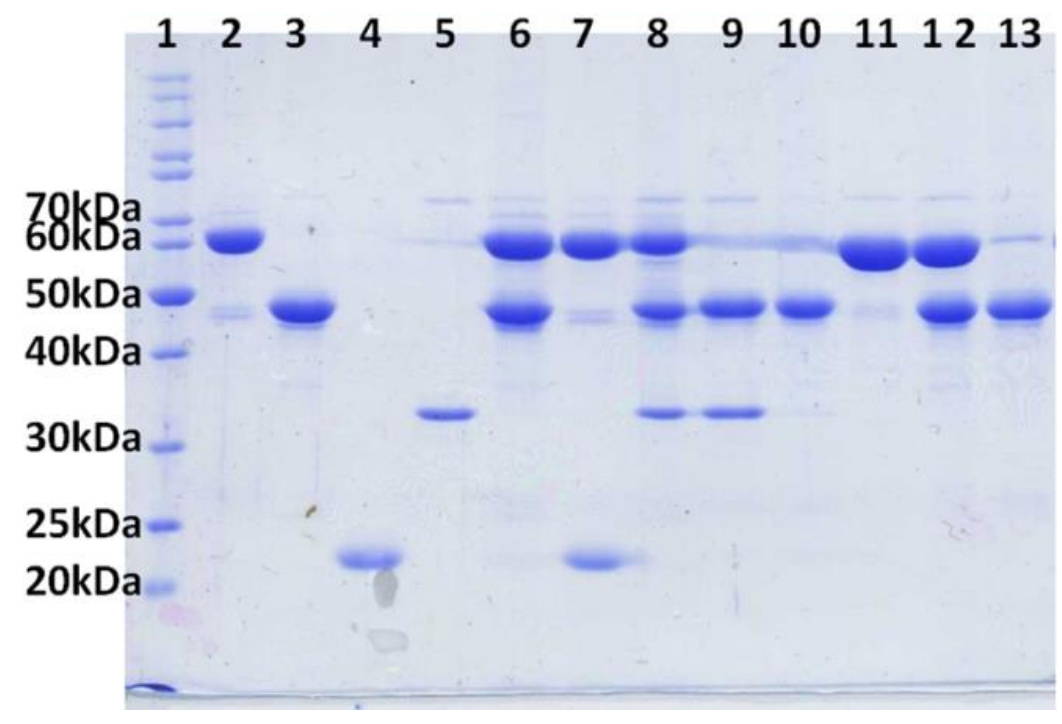

Figure 5. Purified ACAD9, ECSIT, and NDUFAF1 pull-down assay on a Ni-NTA affinity column. Lanes 1. MW Marker, 2. ACAD9-His 6 , 3. ECSIT, 4. C-ECSIT, 5. NDUFAF1, 6. Pull-down product of ACAD9-His ${ }_{6}+$ ECSIT, 7. Pull-down product of ACAD9-His ${ }_{6}+$ C-ECSIT. 8. Pull-down product of

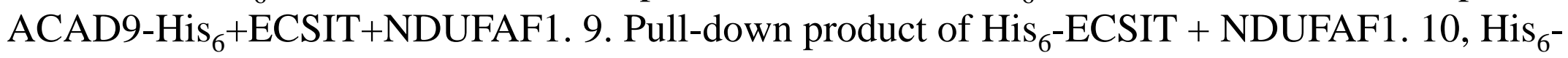
ECSIT, 11. VLCAD, 12. 1:1 mixture of His $_{6}$-ECSIT + VLCAD, 13. Pull-down product of His $_{6}-$ ECSIT +VLCAD. Lanes 6, 7, 8 show that ACAD9-His ${ }_{6}$ can pull down either ECSIT, C-ECSIT, or both ECSIT and NDUFAF1 at the same time. Lane 9 shows His $_{6}$-ECSIT can pull down NDUFAF1. As a control, His $_{6}$-ECSIT failed to pull down VLCAD shown in lane 13. 

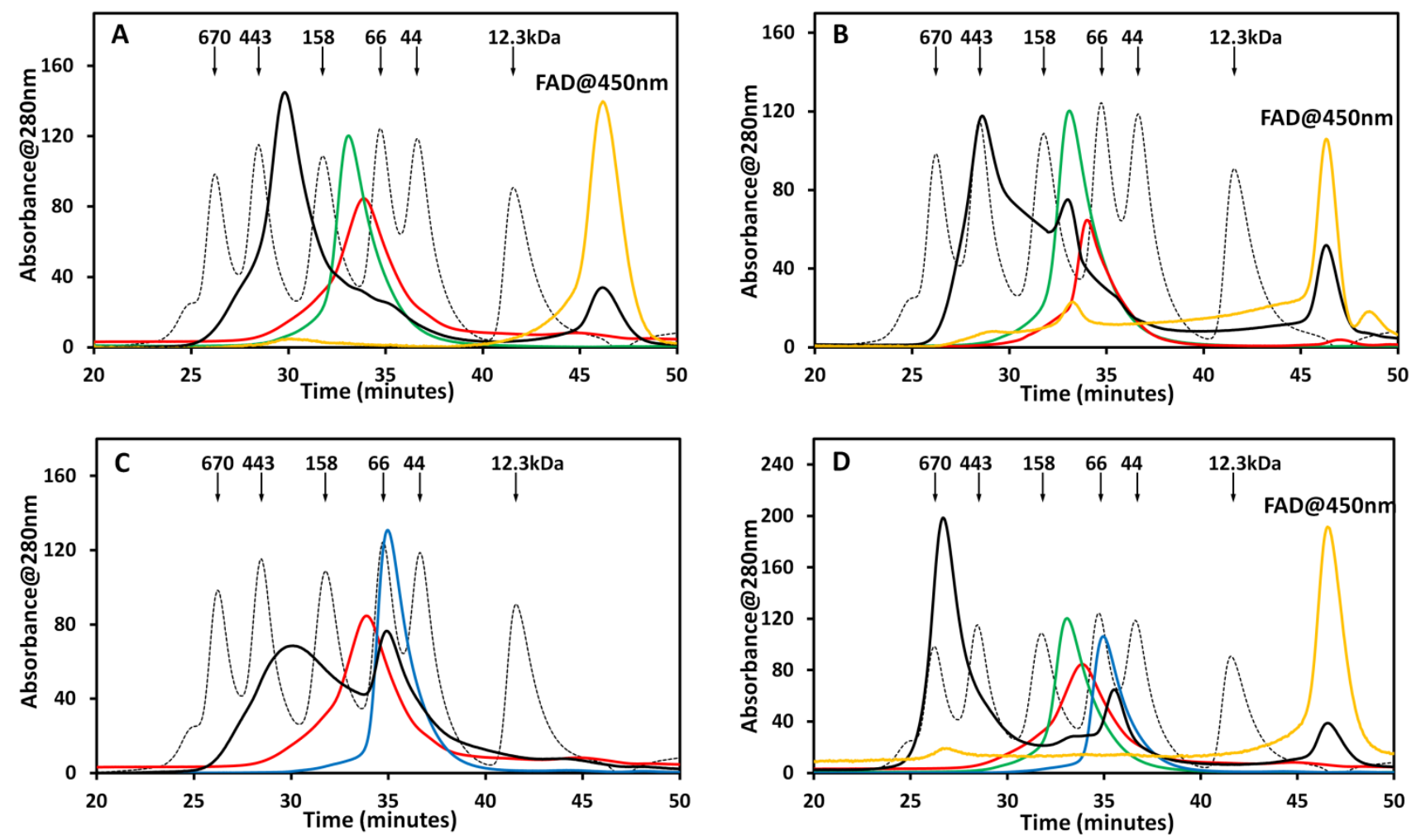

Figure 6. Chromatographic elution profiles showing the complex formation of (A) ACAD9/His ${ }_{6}$-ECSIT, (B)ACAD9/His ${ }_{6}$-C-ECSIT, (C) His ${ }_{6}$ ECSIT/His $_{6}$-SMT3-NDUFAF1, and (D) ACAD9/His 6 -ECSIT/His 6 -SMT3NDUFAF1. ACAD9, His $_{6}$-ECSIT or His $_{6}$-C-ECSIT, and His ${ }_{6}$-SMT3-NDUFAF1 alone are shown in green, red, and blue, respectively. The black and gold profiles are the mixtures of equimolar individual proteins recorded at $280 \mathrm{~nm}$ and 450nm, respectively. For better comparison, the absorbance at 450nm in gold profiles was multiplied by 6 to 10 times. The standard MW protein peaks(grey) from left to right include thyroglobulin (670kDa), apoferritin(443kDa), $\operatorname{IgG}(158 \mathrm{kDa}), \mathrm{BSA}(67 \mathrm{kDa})$, ovalbumin(43kDa), and cytochrome $c(12.3 \mathrm{kDa})$. 
A

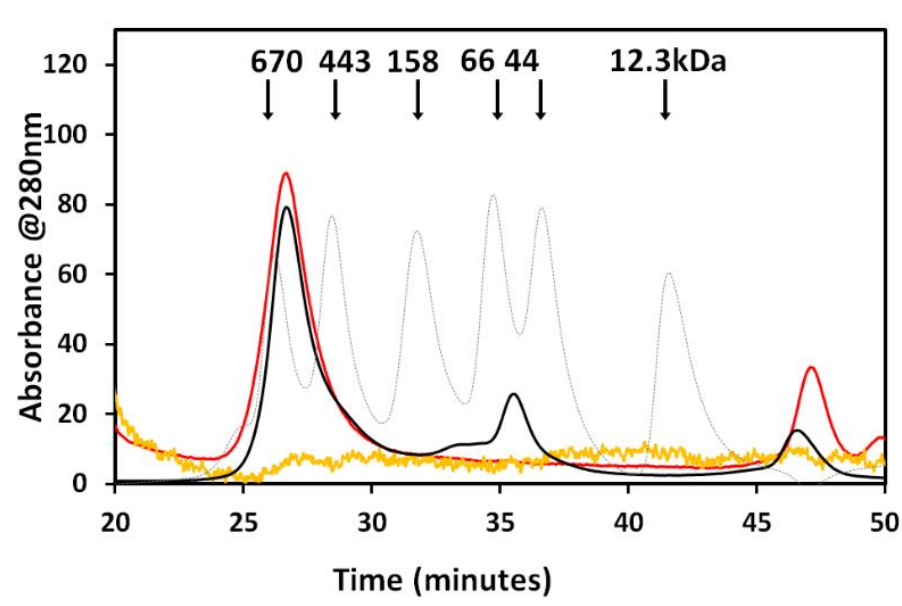

B

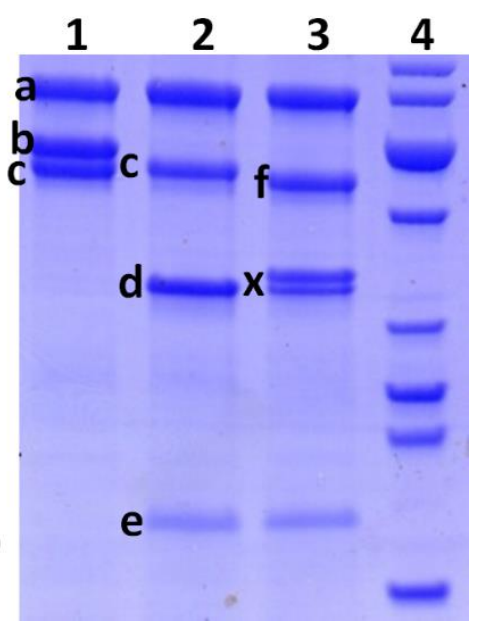

Figure 7. Cleavage of the His6-SMT3 tag from His6-SMT3-NDUFAF1 in ACAD9/His6-ECSIT/His6-SMT3-ACAD9 complex does not change its stability. A) HPLC elution profile of the 1:1:1 mixture of ACAD9/His6-ECSIT/His6-SMT3NDUFAF1 is shown in black (the same profile as in Figure 6D), and the tag-free sample is shown in red. The fractions at 2628 minutes (lane 1 in B) were digested with UPL1 to cleave the His6-SMT3 tag (lane 2 in B) and injected onto the HPLC column. The yellow curve is the HPLC profile at 450nm of the His6-SMT3 tag-cleaved ternary complex (the absorbance value has been scaled up 20 times of the black curve). The small peaks between 45 to 50 minutes in the His6-SMT3 tag-free HPLC profile (red) are from the contaminations rather than FAD due to the small amount of sample injected. The standard MW protein peaks (grey) from left to right include thyroglobulin $(670 \mathrm{kDa})$, apoferritin (443kDa), IgG(158kDa), BSA $(67 \mathrm{kDa})$, ovalbumin $(43 \mathrm{kDa})$, and cytochrome $c(12.3 \mathrm{kDa})$. B) SDS-PAGE of the ACAD9/ECSIT/NDUFAF1 ternary complex. Lane 1, the ternary ACAD9/His ${ }_{6}$ ECSIT/His $_{6}$-SMT3-NDUFAF1 complex purified from HPLC (black curve in A). Lane2, protein in lane 1 was digested overnight by UPL1. This digested product was directly injected onto size exclusion column (red curve in A). Lane 3, protein in lane 1 was digested overnight by both UPL1 and thrombin. Lane 4, protein markers from top to bottom band are 70, 60, 50, 40, 30, 25, 20, $15 \mathrm{kDa}$. Band a, b, c, d, e,f are ACAD9, His6-SMT3NduFAF1, His $_{6}$-ECSIT, NDUFAF1, His $_{6}$-SMT3 tag, and ECSIT, respectively. Double band (X) most likely contains NDUFAF1(lower band) and the product of His6-SMT3-NDUFAF1 cleaved by thrombin due to a non-specific cleavage of the SMT3 tag (upper band). 


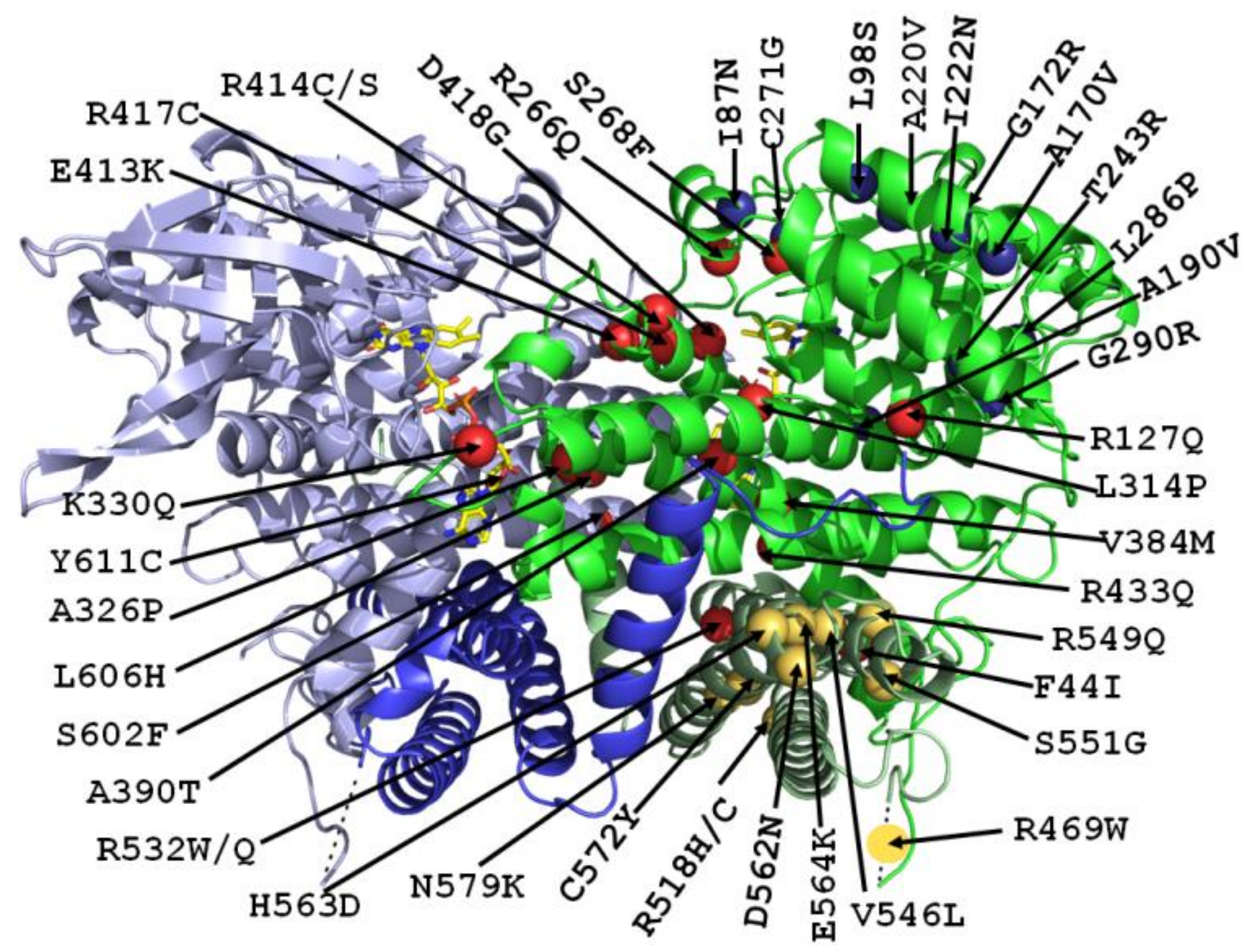

Figure 8. Homology-modeled structure of ACAD9 homodimer with 42 currently known CI deficient mutations marked. The mutations near the surface in the $\mathrm{N}$-terminal domain that might be involved in ECSIT binding are colored blue; the mutations that might decrease the ACAD9 stability including those involved in the dimer interface interactions are colored red; the mutations in the C-terminal domain that are unlikely involved in the dimer interface interactions are colored yellow. 


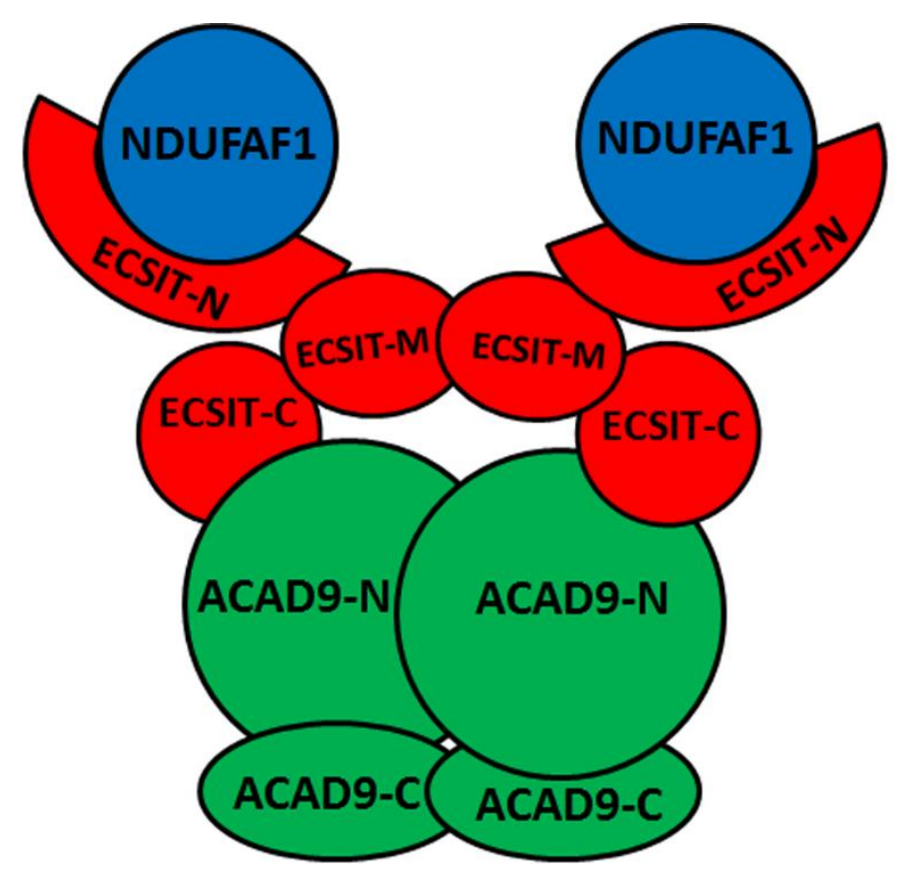

Figure 9. A cartoon model of the ternary complex ACAD9-ECSITNDUFAF1. The ECSIT C-terminal domain binds to the Nterminal domain of ACAD9 and the N-terminal domain of ECSIT binds to NDUFAF1. 